\title{
Gravity Currents in Confined Channels with Environmental Shear
}

\author{
GEORGE H. BRYAN AND RICHARD ROTUNNO \\ National Center for Atmospheric Research,* Boulder, Colorado
}

(Manuscript received 29 May 2013, in final form 17 October 2013)

\begin{abstract}
This study examines properties of gravity currents in confined channels with sheared environmental flow. Under the assumptions of steady and inviscid flow, two-dimensional analytic solutions are obtained for a wide range of shear values. The slope of a gravity current interface just above the surface increases as environmental shear $\alpha$ increases, which is consistent with previous studies, although here it is shown that the interface slope can exceed $80^{\circ}$ for nondimensional shear $\alpha>2$. Then the inviscid-flow analytic solutions are compared with two- and three-dimensional numerical model simulations, which are turbulent and thus have dissipation. The simulated current depths are systematically lower, compared to a previous study, apparently because of different numerical techniques in this study that allow for a faster transition to turbulence along the gravity current interface. Furthermore, simulated gravity current depths are $10 \%-40 \%$ lower than the inviscid analytic values. To explain the model-produced current depths, a steady analytic theory with energy dissipation is revisited. It is shown that the numerical model current depths are close to values associated with the maximum possible dissipation rate in the simplest form of the analytic model for all values of $\alpha$ examined in this study. A primary conclusion is that dissipation plays an important and nonnegligible role in gravity currents within confined channels, with or without environmental shear.
\end{abstract}

\section{Introduction}

Severe thunderstorms often have layers of relatively cold air near the surface (typically called "cold pools") that are created by evaporation and melting of hydrometeors. The relatively cold air usually sinks and spreads along the surface, displacing relatively warmer environmental air that is forced upward. These flow patterns, called gravity currents (or density currents), have been studied analytically for quite some time [see, e.g., Ungarish (2009) for a review]. Theoretical analytic studies pioneered by von Kármán (1940) and Benjamin (1968) are often used to explain the propagation speed of the cold pool's surface gust front given parameters such as the excess density and depth of the relatively cold air.

In a landmark study of the problem, Benjamin (1968) showed how steady gravity current propagation is affected by a finite channel depth (the distance between

\footnotetext{
* The National Center for Atmospheric Research is sponsored by the National Science Foundation.

Corresponding author address: George H. Bryan, NCAR, 3090 Center Green Drive, Boulder, CO 80301.

E-mail: gbryan@ucar.edu
}

flat, rigid lower and upper boundaries) in unsheared environments. Specifically, gravity currents propagate more slowly as channel depth decreases (all else remaining fixed). Benjamin (1968) also showed that if one neglects kinetic-energy dissipation (i.e., if the flow is assumed inviscid) then only one solution is possible: the dense air must fill exactly one-half of the channel at some point behind the surface front where the flow is hydrostatic. When dense air fills less than one-half of the channel depth in this region, steady solutions are still possible, but kinetic-energy dissipation (or simply "dissipation") is required to maintain steady flow-force balance (i.e., conservation of total mass and momentum). From a series of arguments, Benjamin (1968) concluded that dissipation is likely in most cases, and that it plays an "essential role" (Benjamin 1968, p. 247) in gravity current dynamics.

In the atmospheric sciences, several analytic studies have focused on the effects of environmental wind shear on gravity currents, because both shear and cold pools are prevalent in severe storm environments. In one series of articles (Xu 1992, hereafter X92; Xu and Moncrieff 1994; Xue et al. 1997, hereafter XXD97; and Xue 2000) analytic study found, generally speaking, that the depth and propagation speed of the relatively cold air must increase as environmental shear increases to 


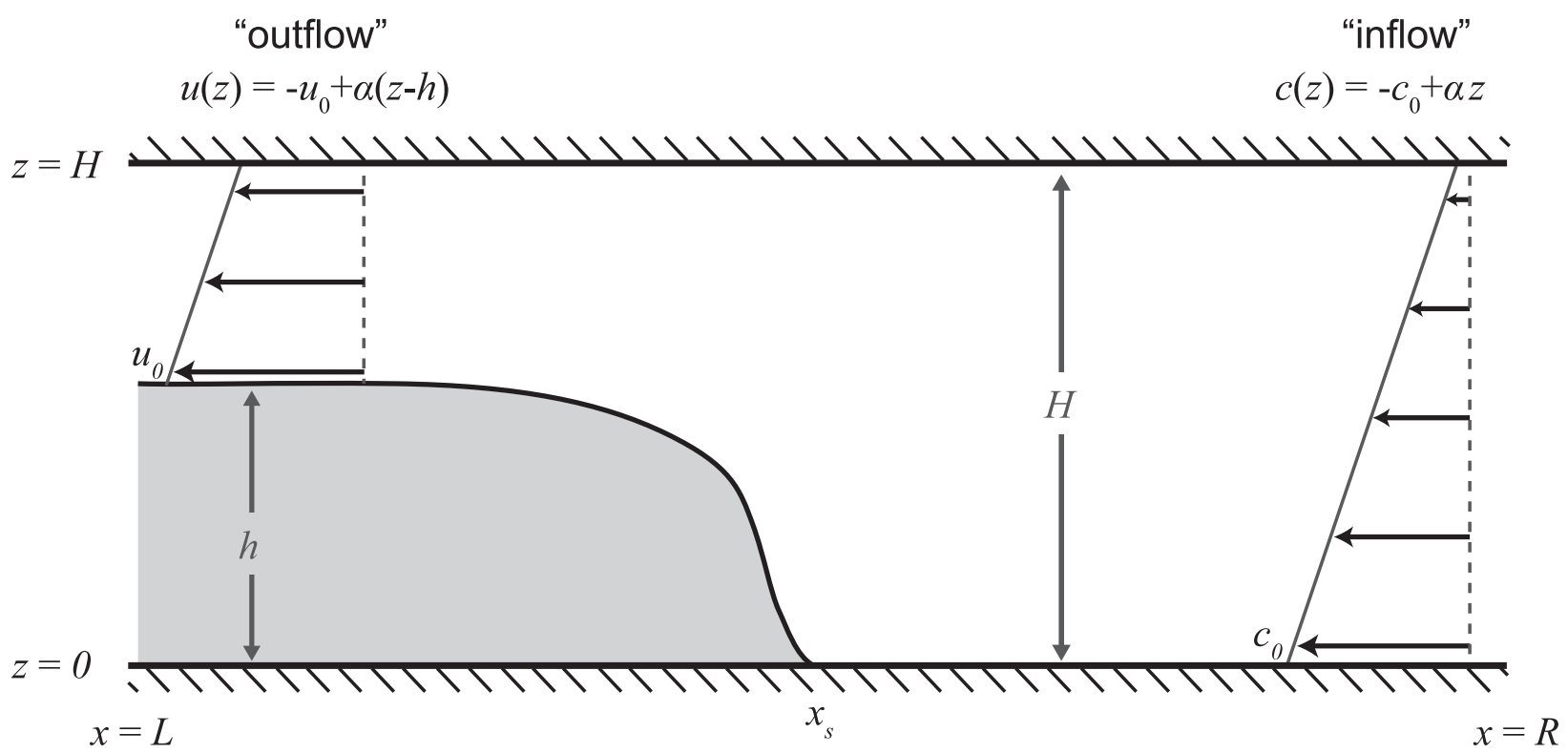

FIG. 1. Conceptual setup for the analytic study of a gravity current in a confined channel with environmental shear. The horizontal wind speed at the bottom-right corner of the control volume is $c_{0}$ and the horizontal wind speed just above the cold pool (i.e., bottom of the outflow) on the left side of the control volume is $u_{0}$. For this study, the flow is assumed stagnant in the relatively dense air (i.e., the cold pool, indicated by gray shading).

maintain steady flow-force balance. In a related series of two-dimensional, time-dependent numerical simulations that were initialized partly with the analytic results, Xu et al. (1996, hereafter XXD96), XXD97, and Xue (2000) showed support for the analytic results, and noted that turbulence was prevalent along gravity current interfaces, which implies the existence of dissipation (e.g., Wyngaard 2010).

A primary goal of the present study is to reexamine how well the inviscid-flow analytic solutions from past studies compare with numerical model simulations. Because of advances in computing technology, we are able to use higher resolution than was used previously, and we can conduct simulations in three dimensions (instead of two, which was used in all the aforementioned studies). These advances allow for a more-realistic treatment of turbulence and its associated dissipation.

We also revisit analytic solutions for gravity currents in shear that account for dissipation (i.e., do not assume inviscid flow). Among the articles cited in previous paragraphs, only X92 (his section 4) studied this problem analytically. We examine the simplest form of his analysis, in which dissipation is uniform with height [the same approach used by Benjamin (1968)], and compare these results with numerical model output. To our knowledge, the following comparison of dissipative analytic results with numerical model output has never been done previously for cases with environmental shear.
The scope of the present study is limited to uniform environmental shear that completely fills a channel, which is the same setup studied by X92 and XXD96. Flow within the cold pool, which was considered by $\mathrm{Xu}$ and Moncrieff (1994) and some later studies, is neglected for simplicity and because such flow has only a small effect in most cases (e.g., Weisman 1992, p. 1840; Xue 2002).

\section{Review of steady, inviscid analytic theory}

In this section we briefly review steady, inviscid analytic theory for gravity currents. The conceptual framework is shown schematically in Fig. 1. The relatively dense air (i.e., cold pool, denoted by shaded region) is assumed to move at speed $c_{0}$ and has an approximately constant depth $h$ at some point behind the surface gust front (which is located at $x_{s}$ ). The gravity current is assumed to exist in a channel of depth $H$. The cold pool is assumed to have constant buoyancy $\hat{b}=-g \Delta \theta / \theta_{0}$ where $g$ is gravitational acceleration, $\theta_{0}$ is the (constant) potential temperature of the environment (which is assumed to be isentropic and thus gravity waves are not considered), and $\Delta \theta$ is the (constant) potential temperature difference across the gravity current interface. A constant value for $\Delta \theta$, and thus $\hat{b}$, is technically not necessary but this assumption is often made for the sake of simplicity. In this framework, a convenient velocity scale $U$ associated with specified parameters $H$ and $\hat{b}$ is defined as follows: $U \equiv(\hat{b} H)^{1 / 2}$. 
TABLE 1. Maximum values of nondimensional gravity current depth $h / H$ for specified environmental shear $\alpha$. The first row lists analytic values for steady inviscid flow (X92, section 2 ). The second row lists two-dimensional numerical model results from XXD96 (their Table 1). All remaining results are from numerical simulations in the present study, analyzed over $-3 \leq x / H \leq 0$, using timeaveraged output from 20 to $30 \mathrm{~min}$

\begin{tabular}{lcccc}
\hline \multicolumn{1}{c}{ Simulations } & $\alpha=-0.84$ & $\alpha=0$ & $\alpha=0.88$ & $\alpha=2.26$ \\
\hline Inviscid analytic & 0.300 & 0.500 & 0.700 & 0.900 \\
2D from XXD96 with & 0.214 & 0.405 & 0.667 & 0.857 \\
$\quad \begin{array}{c}\Delta x=50 \mathrm{~m}, \Delta z=25 \mathrm{~m} \\
\Delta x=50 \mathrm{~m}, \Delta z=25 \mathrm{~m}\end{array}$ & 0.187 & 0.356 & 0.635 & 0.827 \\
$\Delta x=\Delta z=25 \mathrm{~m}$ & 0.191 & 0.364 & 0.583 & 0.826 \\
$\Delta x=\Delta z=12.5 \mathrm{~m}$ & 0.200 & 0.370 & 0.588 & 0.813 \\
$\Delta x=\Delta z=6.25 \mathrm{~m}$ & 0.205 & 0.366 & 0.602 & 0.829 \\
$\Delta x=\Delta z=3.125 \mathrm{~m}$ & 0.209 & 0.372 & 0.593 & 0.828 \\
\hline
\end{tabular}

The Boussinesq approximation is made in this study, which is applicable to atmospheric gravity currents having depth $h$ that is small compared to the density scale height $(\sim 8 \mathrm{~km})$. Free-slip boundary conditions are assumed for the rigid lower and upper boundaries. Finally, an assumption made for this section only is that the flow is inviscid.

\section{a. Flow-force balance}

By integrating the governing equations for mass and momentum over the entire volume shown in Fig. 1, it is possible to determine required relationships between variables such as current depth $h$ and propagation speed $c_{0}$, assuming the flow remains steady. This analysis is done in a frame of reference moving with the surface gust front. The environmental flow (i.e., the flow on the right side of the control volume) is assumed herein to have form $c(z)=-c_{0}+\alpha z$ for $0 \leq z \leq H$; that is, the incoming flow varies linearly with height over the entire channel. The parameter $\alpha$, which is the vorticity in the environment, is used herein to quantify the environmental shear, as in X92.

Results for $\alpha \neq 0$ were first obtained by X92; further details can be found in his section 2. Under the assumptions noted above, for any specified shear $\alpha$ there is only one solution for nondimensional current depth $h / H$ and propagation speed $c_{0} / U$. For $\alpha=0$ (no environmental shear) these values are $h / H=c_{0} / U=0.5$, which were first determined by Benjamin (1968). The primary discovery by $\mathrm{X} 92$ is that both $h / H$ and $c_{0} / U$ must increase as $\alpha$ increases to maintain steady, inviscid flow-force balance. Some values of these two variables are listed in the first rows of Tables 1 and 2.

\section{b. Interior flow solutions}

Details of the interior flow (i.e., the flow within the control volume) can also be determined under the
TABLE 2. As in Table 1, but for nondimensional propagation speed $c_{0} / U$.

\begin{tabular}{lcccc}
\hline \hline \multicolumn{1}{c}{ Simulations } & $\alpha=-0.84$ & $\alpha=0$ & $\alpha=0.88$ & $\alpha=2.26$ \\
\hline Inviscid analytic & 0.328 & 0.500 & 0.757 & 1.252 \\
2D from XXD96 with & 0.318 & 0.486 & 0.722 & 1.30 \\
$\quad \Delta x=50 \mathrm{~m}, \Delta z=25 \mathrm{~m}$ & & & & \\
$\quad \Delta x=50 \mathrm{~m}, \Delta z=25 \mathrm{~m}$ & 0.297 & 0.478 & 0.746 & 1.241 \\
$\Delta x=\Delta z=25 \mathrm{~m}$ & 0.296 & 0.473 & 0.745 & 1.246 \\
$\Delta x=\Delta z=12.5 \mathrm{~m}$ & 0.297 & 0.476 & 0.741 & 1.241 \\
$\Delta x=\Delta z=6.25 \mathrm{~m}$ & 0.298 & 0.477 & 0.741 & 1.241 \\
$\Delta x=\Delta z=3.125 \mathrm{~m}$ & 0.298 & 0.477 & 0.741 & 1.240 \\
\hline
\end{tabular}

assumptions of steady and inviscid flow. The key step in this type of analysis is to determine the shape of a gravity current interface $h(x)$ such that Bernoulli energy is constant along the lowermost streamline in the flow (i.e., the streamline that passes along the surface for $x>$ $x_{s}$ and along the top of the cold pool for $x<x_{s}$ ). The interior flow must also satisfy the equation $\nabla^{2} \psi=\alpha$ (i.e., the definition of vorticity), where $\psi$ is streamfunction. Details can be found in section 3 of X92.

To our knowledge, precise solutions for $h(x)$ have only been determined numerically. X92 used an iterative method on a finite-element grid, as described by $\mathrm{Xu}$ et al. (1992). The control-volume solutions for maximum height $h_{m}$ and propagation speed $c_{0}$ (see previous subsection) are used as lateral boundary conditions. Results by X92 (his Fig. 4) reveal two important conclusions that we evaluate further in this study: 1) the slope of the interface must be $60^{\circ}$ at the surface, regardless of the value of $\alpha$ (X92, p. 515), and 2) the slope of the interface above the surface (e.g., at $\left.z=0.5 h_{m} / H\right)$ increases as $\alpha$ increases (X92, p. 515).

Here we reproduce the results of X92, and we also consider larger values of $\alpha$ than were shown in his study. To this end, we created two-dimensional solutions that generally follow the technique of $\mathrm{Xu}$ et al. (1992). Our overall approach to determine $h(x)$ is essentially the same; that is, we iteratively adjust the lower surface of an analysis grid, assuming that this lower surface represents the top of a gravity current cold pool. The most notable difference from the methodology of Xu et al. (1992) is that we use a grid with strictly vertical columns, rather than the more general finite-element mesh used by Xu et al. (1992). We further use the coordinate transform of Gal-Chen and Somerville (1975), such that the shape of the gravity current interface is handled equivalently to terrain in many atmospheric numerical models. The solution procedure is nearly identical to Xu et al. (1992) except that our "guesses" for new current depth at each iteration are simple additions or subtractions of a small value, with the sign of the displacement based on the diagnosed value of Bernoulli energy along the lowermost 

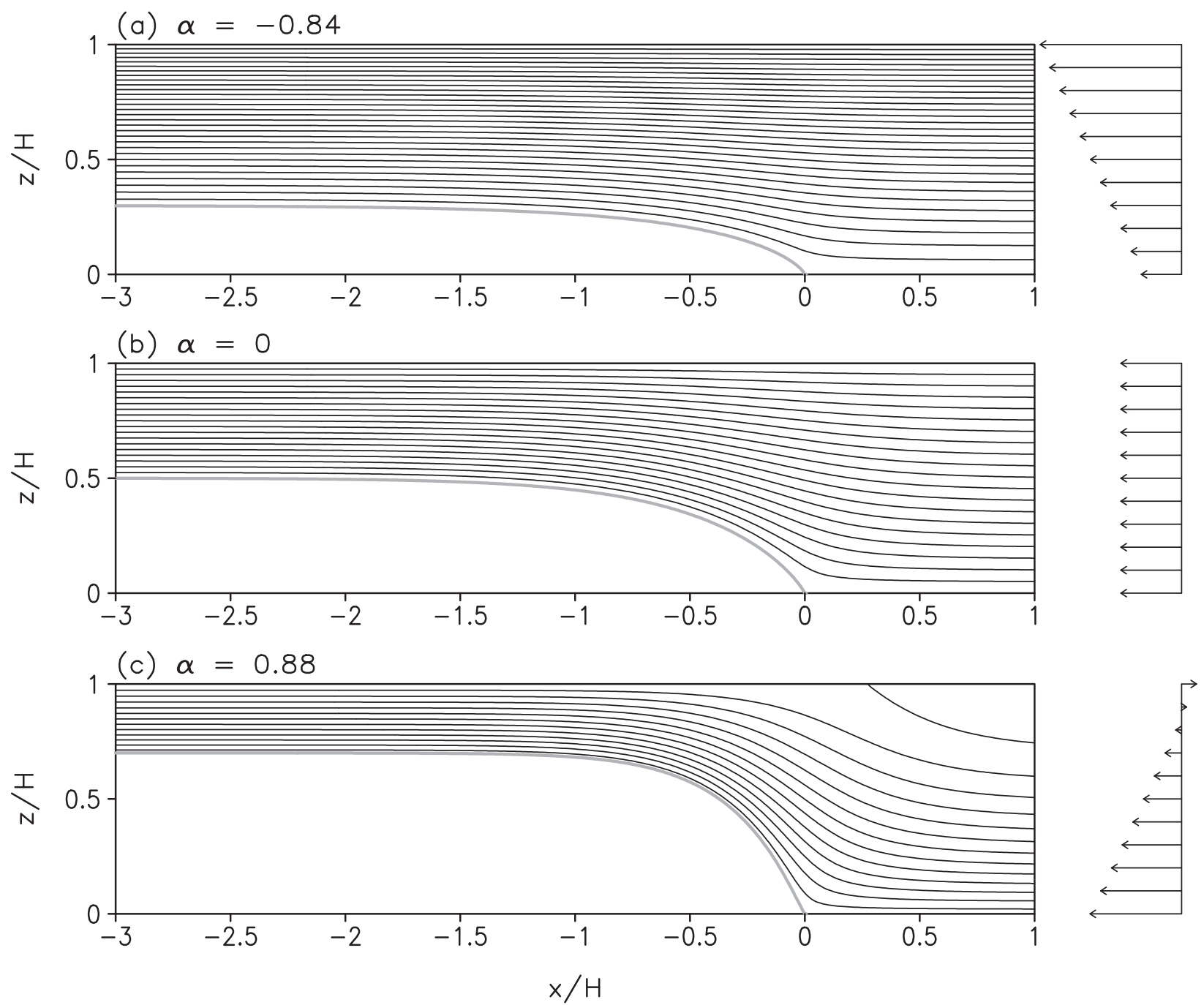

FIG. 2. (left) Current interface (gray contour) and nondimensional streamfunction (black contours every 0.025), and (right) environmental wind profile (vectors), for steady inviscid flow with $\alpha=$ (a) -0.84 , (b) 0 , and (c) 0.88 (cf. Fig. 4 of X92). A vector length of $1 H$ corresponds to a vector magnitude of $1.5 \mathrm{U}$.

streamline. Our iteration method is cruder than the one used by Xu et al. (1992), and undoubtedly requires more iterations, but solutions with errors in $h$ less than $0.001 H$ are determined in a few minutes on a single-processor workstation using a $1601 \times 161$ analysis grid.

Results for three different values of environmental shear $\alpha$ are shown in Fig. 2. The close correspondence between this figure and an analogous one by X92 (his Fig. 4) demonstrates the ability of our approach to reproduce his results.

Further results-including solutions for relatively large environmental shear not considered by X92-are shown in Fig. 3. Variables for this figure have been dimensionalized using the scaling parameters of XXD96 (channel depth $H=1 \mathrm{~km}$, velocity scale $U=10 \mathrm{~m} \mathrm{~s}^{-1}$, potential temperature difference $\Delta \theta=-3 \mathrm{~K}$ ).
For Fig. 3a, there is no environmental shear $(\alpha=0)$. Our interface (gray curve) is indistinguishable from that determined by Benjamin (1968, his Fig. 11). The inset shows the expected result at the surface (i.e., a $60^{\circ}$ angle). Figure $3 \mathrm{~b}$ shows the highest-shear case examined by X92, $\alpha=0.88$. As noted by $\mathrm{X} 92$, the current interface has roughly a $60^{\circ}$ angle from the surface to approximately $0.5 h_{m} / H$. For Fig. $3 \mathrm{c}$, the difference in wind speed $\Delta U$ across the domain in the environment matches the integrated cold-pool intensity $C$, determined by the equation $C^{2}=2 \int_{0}^{h} \hat{b} d z$ (e.g., Rotunno et al. 1988). In this case, the expected $60^{\circ}$ angle is found at the surface, but the interface is notably steeper than $60^{\circ}$ just away from the surface. For Fig. 3d, we show the "high shear" case of XXD96 $(\alpha=2.26)$. The interface slope exceeds $80^{\circ}$ in this case. 

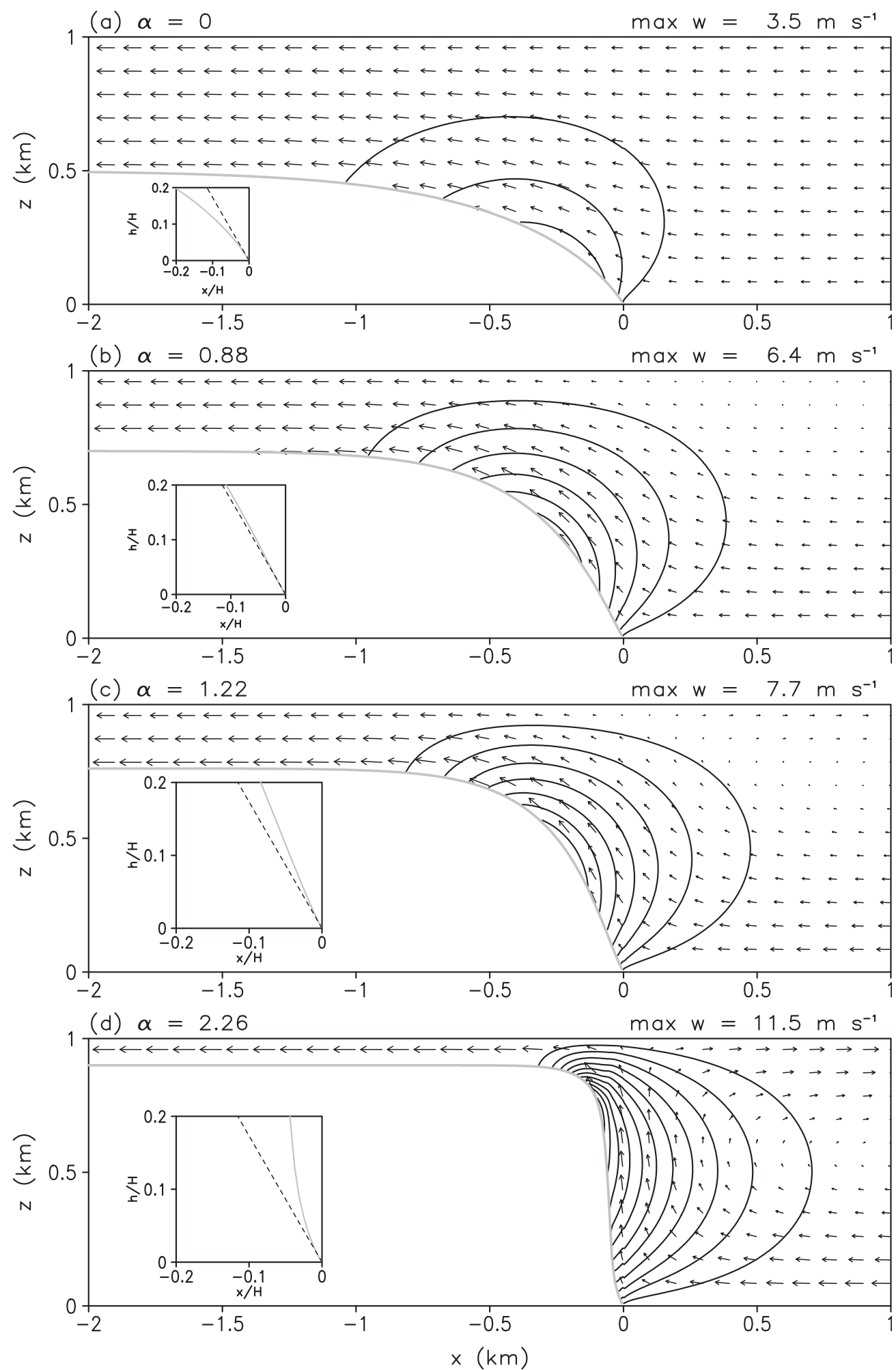

FIG. 3. Steady inviscid gravity current solutions for environmental shear $\alpha=$ (a) 0 , (b) 0.88 , (c) 1.22 , and (d) 2.26. Gray contour is the interface, black contours show vertical velocity every $1 \mathrm{~m} \mathrm{~s}^{-1}$, and vectors show surface-front-relative flow where a vector length of $0.1 \mathrm{~km}$ represents a vector magnitude of $15 \mathrm{~m} \mathrm{~s}^{-1}$. The inset in the lower-left corner shows a close-up view of the interface near the surface; the dashed line denotes a $60^{\circ}$ angle from the horizontal. 
The results in Fig. 3 reconfirm many conclusions from previous studies. For example, the interface slope at the surface is $60^{\circ}$, regardless of the value for $\alpha$ (e.g., Rottman et al. 1985, p. 279; X92, p. 515). Also, as environmental shear increases, the gravity current just behind the surface gust front is "thicker" (Rottman et al. 1985, p. 273) or “deeper" (X92, p. 515); as noted by X92 (p. 515), this result implies that interface slope above the surface (e.g., at $0.5 h_{m} / H$ ) becomes steeper as shear increases. And, for large shear $(\alpha \gtrsim 1)$, the interface slope at one-half the maximum height $\left(h_{m}\right)$ exceeds $60^{\circ}(\mathrm{Xu}$ and Moncrieff 1994, p. 443).

In the sections that follow, we evaluate whether numerical model simulations reproduce the analytic solutions discussed in this section. We note that inviscid flow has been assumed throughout this section but that timedependent numerical models of atmospheric flows typically (if not always) include viscous effects.

\section{Numerical modeling methodology}

\section{a. The numerical model}

The numerical model for this study uses the compressible Boussinesq equations,

$$
\begin{aligned}
& \frac{\partial u}{\partial t}=-u \frac{\partial u}{\partial x}-v \frac{\partial u}{\partial y}-w \frac{\partial u}{\partial z}-\frac{\partial \phi}{\partial x}, \\
& \frac{\partial v}{\partial t}=-u \frac{\partial v}{\partial x}-v \frac{\partial v}{\partial y}-w \frac{\partial v}{\partial z}-\frac{\partial \phi}{\partial y}, \\
& \frac{\partial w}{\partial t}=-u \frac{\partial w}{\partial x}-v \frac{\partial w}{\partial y}-w \frac{\partial w}{\partial z}-\frac{\partial \phi}{\partial z}+g \frac{\theta^{\prime}}{\theta_{0}}, \\
& \frac{\partial \theta^{\prime}}{\partial t}=-u \frac{\partial \theta}{\partial x}-v \frac{\partial \theta}{\partial y}-w \frac{\partial \theta}{\partial z}, \quad \text { and } \\
& \frac{\partial \phi}{\partial t}=-c_{s}^{2}\left(\frac{\partial u}{\partial x}+\frac{\partial v}{\partial y}+\frac{\partial w}{\partial z}\right),
\end{aligned}
$$

where $u, v$, and $w$ are the three components of velocity, $\theta_{0}$ is a constant reference potential temperature, $\theta^{\prime} \equiv$ $\theta-\theta_{0}$ is perturbation potential temperature, $g$ is gravitational acceleration, $\phi \equiv p^{\prime} / \rho_{0}$ is the pressure variable, $\rho_{0}$ is a constant reference density, and $c_{s}$ is the speed of sound (set to a constant $300 \mathrm{~m} \mathrm{~s}^{-1}$ herein). The perturbation pressure, $p^{\prime} \equiv p-p_{0}$, is defined relative to a reference profile $p_{0}(z)$ that is determined from the hydrostatic equation, $\partial p_{0} / \partial z=-\rho_{0} g$. These equations, in which compressibility is retained and the Boussinesq approximation is made for the pressure-gradient terms, have been used in several other numerical modeling studies of gravity currents (e.g., Droegemeier and Wilhelmson 1987; Klemp et al. 1994; XXD96).
The model is integrated using the three-step RungeKutta scheme, with the split-explicit method for compressible flows, following Wicker and Skamarock (2002). Advection terms are calculated using the fifth-order scheme from their study, except advection for $\theta^{\prime}$ is calculated using a weighted essentially nonoscillatory (WENO) scheme (Shen and Zha 2010). All other gradients are calculated using a second-order centered scheme for the standard staggered $\mathrm{C}$ grid.

Although there are no explicit viscous terms in the model equations, viscous effects do occur in our simulations via the odd-ordered numerical algorithm used for advection. As explained later, the odd-ordered scheme has implicit diffusion of form $\nabla^{6}$ with a diffusion coefficient proportional to Courant number (e.g., Wicker and Skamarock 2002, p. 2089). We decided to neglect explicit diffusion terms based on a similar decision by XXD96 (although they used explicit $\nabla^{4}$ terms for numerical reasons) and because this model setup produces the minimum necessary dissipation for this modeling system.

\section{b. Model setup and initial conditions}

Except for one difference (explained below) we use the same initial conditions as XXD96. In short, the initial interface is a simple mathematical relation [XXD96's Eq. (3.3)] that features a $60^{\circ}$ angle from the horizontal at the surface and roughly approximates the shape of energy-conserving solutions. A comparison of precise inviscid interfaces from section $2 \mathrm{~b}$ with the initial interfaces using XXD96's Eq. (3.3) is shown in Fig. 4. (We choose to use the same initial interfaces as XXD96, rather than the solutions from section 2 herein, to allow for straightforward comparison with their previously published results.) The initial two-dimensional (2D) flow field satisfies the equation $\nabla^{2} \psi=\alpha$ at all points, and the initial 2D pressure $(\phi)$ field is determined from the equation $\nabla^{2} \phi=\partial F_{u_{i}} / \partial x_{i}$, where $F_{u_{i}}(i=1,2,3)$ represents the right-hand sides of Eqs. (1)-(3) (excluding the pressure gradient terms).

The one difference we made, compared to XXD96, is to extend the initial block of cold air all the way to the left boundary, thereby eliminating the need to consider a left-moving front. Because we use open lateral boundary conditions, it is straightforward to allow cold air at the left boundary. We have also conducted all 2D simulations with the exact XXD96 initial state (i.e., cold air in the middle part of the domain only, with both left-moving and right-moving fronts) and found very similar results to those shown herein.

For nondimensionalization, we use the same scaling parameters as XXD96. For reference, they are as follows: the length scale $H$ is $1 \mathrm{~km}$, the velocity scale $U \equiv(H \hat{b})^{1 / 2}$ is $10 \mathrm{~m} \mathrm{~s}^{-1}$, the time scale $T \equiv H / U$ is $100 \mathrm{~s}$, and the potential temperature difference $\Delta \theta$ is $-3 \mathrm{~K}$. All 

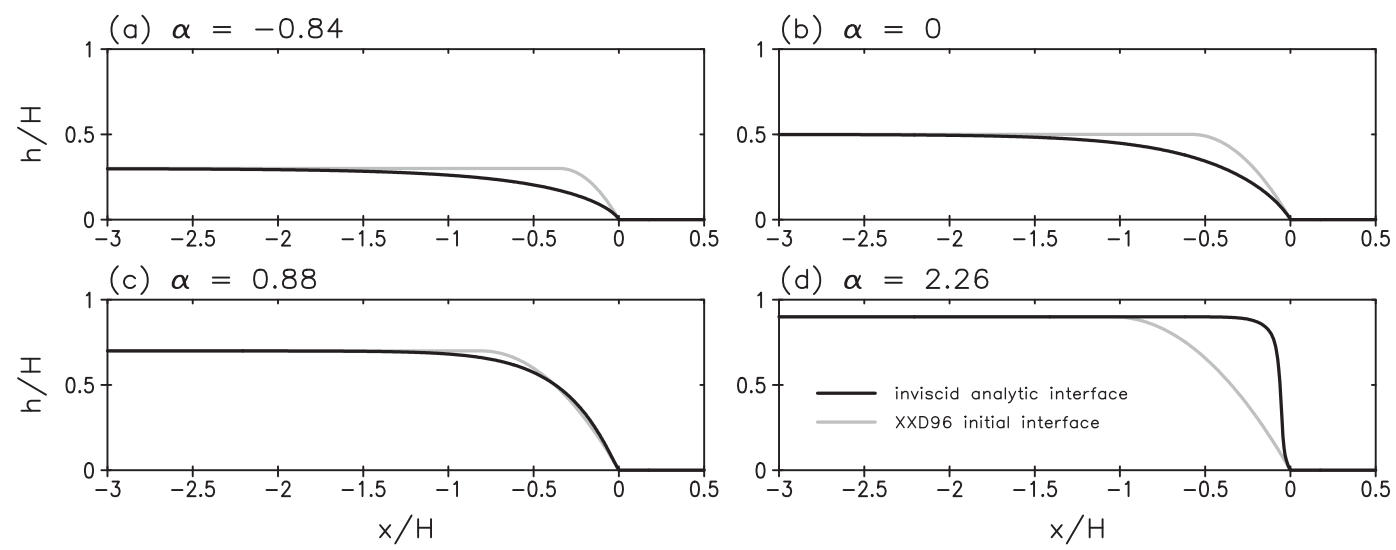

FIG. 4. Comparison of steady, inviscid analytic interfaces (black, from section $2 b$ ) with the approximate interfaces used for initial conditions following XXD96 [gray, their Eq. (3.3)] for $\alpha=$ (a) -0.84, (b) 0, (c) 0.88, and (d) 2.26.

results are presented nondimensionally using these scaling parameters. We set $\rho_{0}=1 \mathrm{~g} \mathrm{~kg}^{-1}$ and $\theta_{0}=300 \mathrm{~K}$.

The domain size is $40 \mathrm{~km}$ in the direction perpendicular to the gravity current front and is $1 \mathrm{~km}$ deep (the same size as in XXD96). For three-dimensional (3D) simulations, the domain size parallel to the initial front is $1 \mathrm{~km}$ (i.e., the same as the channel depth), which is consistent with our previous work (Rotunno et al. 2011). We show the sensitivity to grid spacing in upcoming sections. Freeslip boundary conditions are used at the bottom and top of the domain, and open boundary conditions are used on the left and right sides of the domain. For 3D simulations, periodic boundary conditions are used in the $y$ direction. Random small-amplitude perturbations in $u$ and $v$ are added along the interface in the initial conditions of 3D simulations to initiate 3D motions. All simulations are integrated to at least $1800 \mathrm{~s}$ (18T). Following XXD96, we study four nominal values for environmental shear: $\alpha=$ $-0.84,0,0.88$, and 2.26 , corresponding to steady, inviscid gravity current depths of $h / H=0.3,0.5,0.7$, and 0.9 .

\section{c. Analysis techniques}

Model output is saved every $15 \mathrm{~s}(0.15 T)$. For timeaverage plots, the output is first shifted horizontally so that the surface gust front is in the same location, which is here denoted as $x / H=0$. The location of the surface gust front is defined as the $\Delta \theta=-1.5 \mathrm{~K}$ contour at the lowest model level (i.e., one-half the initial potential temperature difference).

Gravity current depth $h$ is determined quantitatively using the formula

$$
h(x, y)=\frac{1}{\hat{b}} \int_{0}^{H} B(x, y, z) d z,
$$

where $B(x, y, z)=g\left[\theta(x, y, z)-\theta_{0}\right] / \theta_{0}$. This method was also used by Crook and Miller (1985), Klemp et al.
(1994), and Bryan and Rotunno (2008, hereafter BR08), and usually yields similar values to the method of XXD96 (who used the $0.5 \times \hat{b}$ contour). An advantage of Eq. (6), compared to XXD96's method, is that Eq. (6) always yields single-valued results.

\section{Two-dimensional simulations}

We first examine results from 2D simulations, which were used for all simulations by XXD96. The primary goals of this section are to reexamine their results in light of different numerical techniques and higher resolution, and to assess how well the inviscid analytic solutions (section 2 of X92 and section 2 herein) compare with simulated gravity currents.

Table 1 lists maximum values of nondimensional gravity current depth $h / H$; the first row lists the inviscid analytic solutions (which are used in the construction of the initial conditions following XXD96); the second row lists the results from the $2 \mathrm{D}$ numerical simulations of XXD96 (as reported in their Table 1); all other results are from our numerical simulations, where we have averaged $h$ from 20 to $30 \mathrm{~min}$ and list the maximum value from $-3 \leq x / H \leq 0$. The most notable conclusion drawn from Table 1 is that all model simulations (including those by XXD96) produce lower values of $h / H$ than the steady inviscid analytic results of X92. This difference is almost certainly attributable to kinetic-energy dissipation, which seems inevitable in Boussinesq gravity currents because turbulence develops on the sheared current interface (e.g., XXD96, p. 784). Output from 2D simulations for $\alpha=0$ and 2.26 are shown in Figs. 5 and 6. For the highest resolution used in this study $(\Delta x=\Delta z=$ $3.125 \mathrm{~m}$; Figs. 5a and 6a) turbulent eddies clearly develop near the leading edge $(x / H \approx-0.2)$ of the gravity current. 

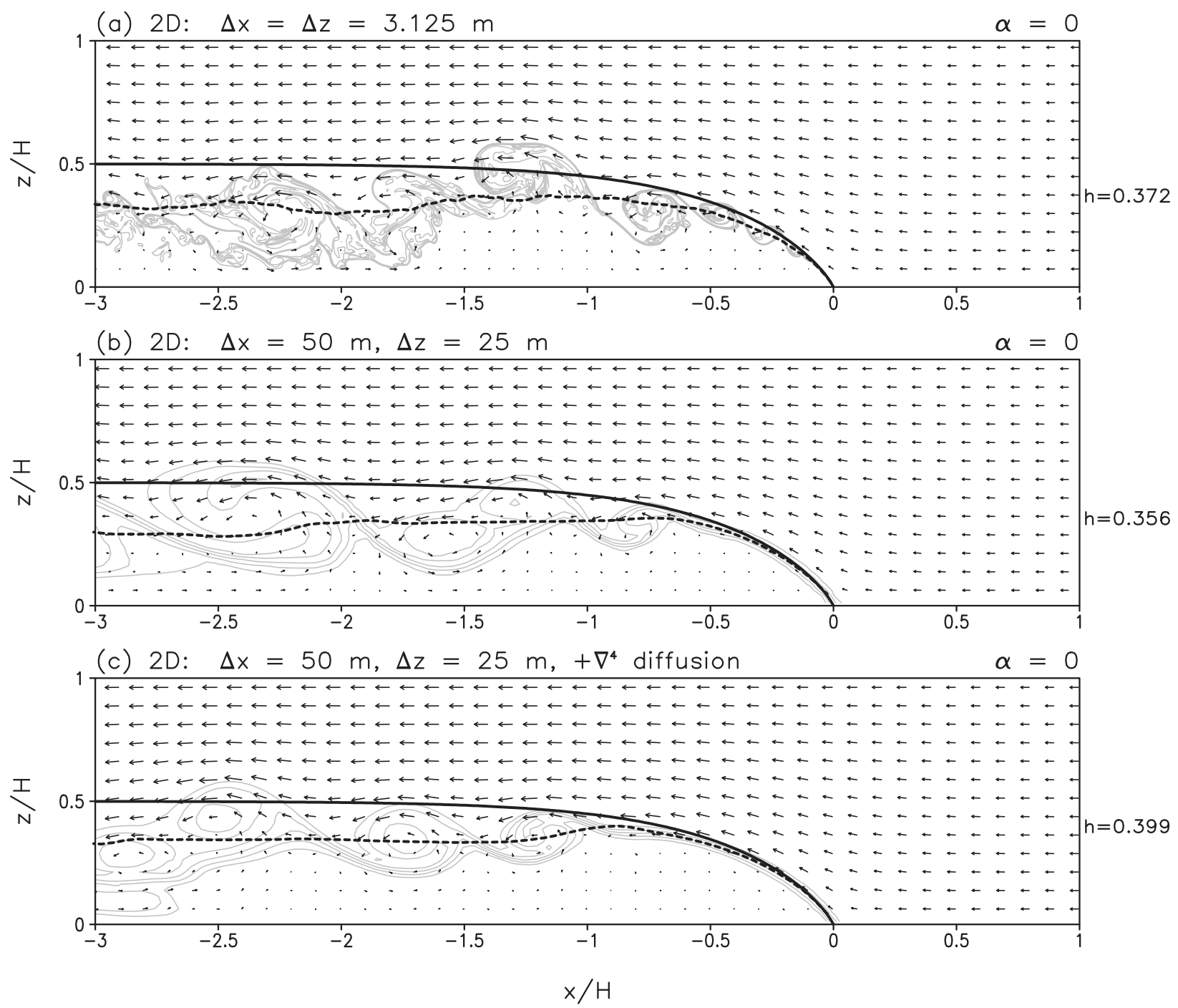

FIG. 5. Two-dimensional numerical model simulations for $\alpha=0$ (no environmental shear). Vectors show gust-front-relative flow at $t=$ $30 \mathrm{~min}$ and gray contours show potential temperature every $0.5 \mathrm{~K}$ at the same time. The dashed black contour shows the diagnosed gravity current depth averaged from 20 to $30 \mathrm{~min}$; the number on the right side of each panel is the maximum value. The solid black contour is the analytic interface for steady inviscid flow. Using (a) $\Delta x=\Delta z=3.125 \mathrm{~m}$; (b) $\Delta x=50 \mathrm{~m}$ and $\Delta z=25 \mathrm{~m}$ (as in XXD96); (c) as in (b), except $\nabla^{4}$ diffusion is included following XXD96.

A second notable result from Table 1 is that all simulations from the present study produce lower values of $h / H$ than the numerical simulations of XXD96. To test whether this difference was attributable to resolution, we repeated our simulations using the same grid spacing as XXD96 $(\Delta x=50 \mathrm{~m}, \Delta z=25 \mathrm{~m})$; these simulations (Figs. 5b and 6b) still produce lower values of $h / H$ than reported by XXD96. We then modified the numerical techniques of our model to more closely match those of XXD96. Ultimately, we found that the $\nabla^{4}$ diffusion scheme of XXD96 inhibits the growth of turbulence along the gravity current interface. For example, with their diffusion scheme (and their settings for diffusion coefficients) added to our model, the turbulent eddies are only apparent away from the surface gust front (i.e., for $x / H \leq-1$ ) (Figs. $5 c$ and $6 c$ ); the same qualitative results can be seen in the figures of XXD96. Maximum values of $h / H$ from these simulations (see values listed on right side of Figs. 5c and 6c) are more comparable to values reported by XXD96. Hence, there is some ambiguity in gravity current simulations due to numerical techniques, with the comparatively higher-order schemes (and flowdependent diffusion) in our model yielding faster-growing turbulence and smaller values of current depth $h / H$.

The no-shear case $(\alpha=0)$ is particularly interesting because it is the case studied in detail by Benjamin 


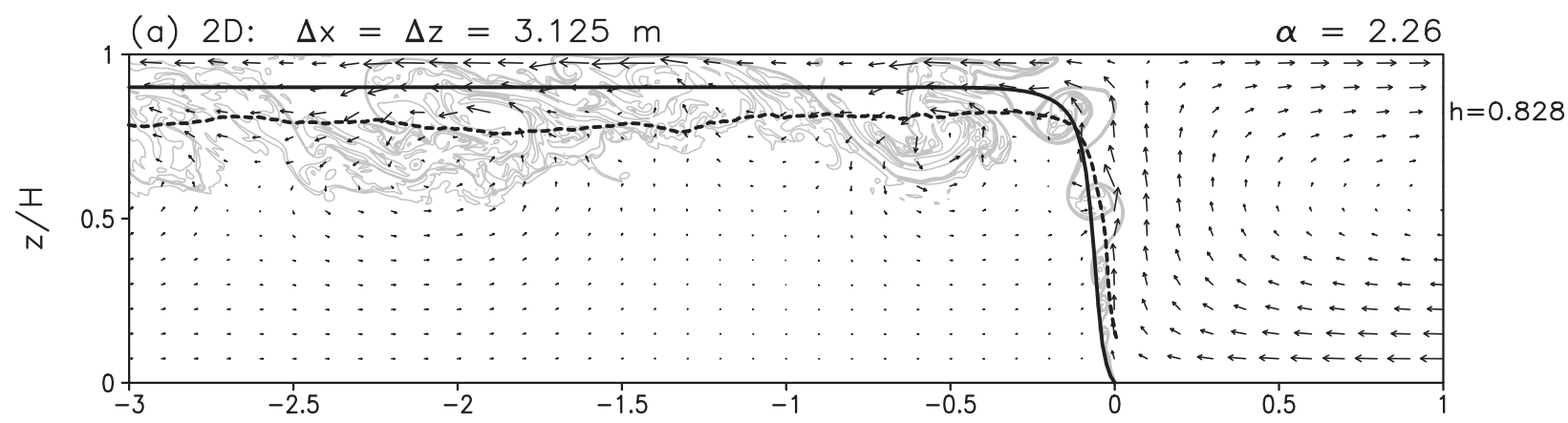

(b) 2D: $\Delta x=50 \mathrm{~m}, \Delta z=25 \mathrm{~m} \quad \alpha=2.26$
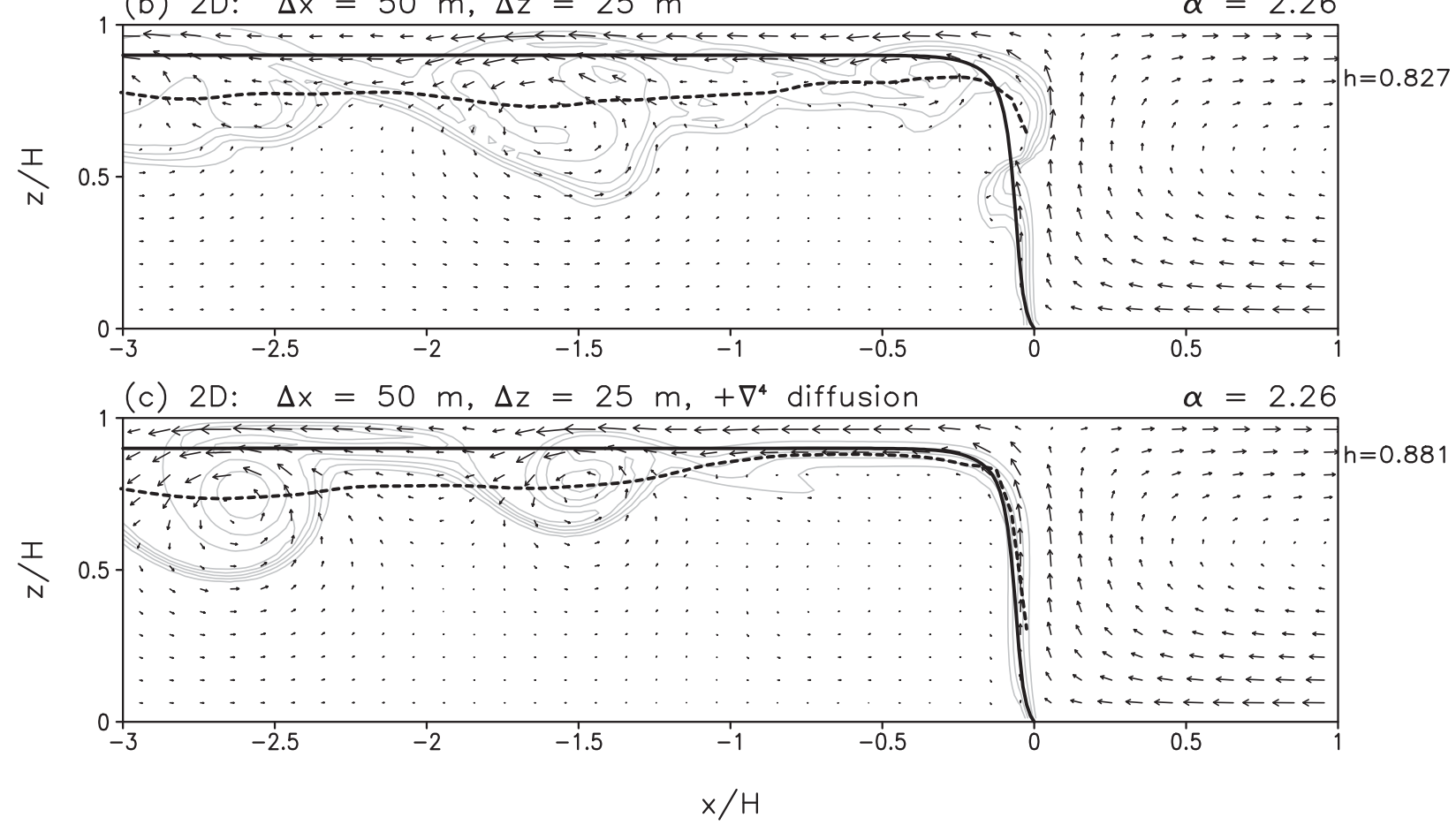

FIG. 6. As in Fig. 5, but for $\alpha=2.26$ (strong environmental shear).

(1968), who concluded that $h / H>0.347$ would be "difficult, if not impossible to produce" (p. 219) because of the likely effects of turbulence, and thus energy dissipation, on the highly sheared current interface. Our model results (Table 1) support this conclusion in the sense that current depths are much closer to 0.347 (the value corresponding to maximum possible dissipation rate) than to 0.5 (the inviscid value) (Table 1). If we calculate $h / H$ at a distance far enough behind the gravity current for the flow to be hydrostatic (typically $x / H<$ $-1.5)$ then $h / H$ is less than 0.347 for all simulations.

Figures 5 and 6 also show, as thick black lines, the analytic current interfaces for steady, inviscid flow (see section $2 b$ ). The model simulations only conform to this shape very close to the surface where turbulent eddies are necessarily weak. Once the turbulence develops, and mixing occurs between the cold and warm fluid, then the simulated current interface departs from the inviscid analytic interface. For the zero-shear case, this departure happens sooner (i.e., closer to the surface) with higher resolution (cf. Figs. 5a and 5b). Furthermore, we note that for heights between 0.35 and 0.5 , the mean flow is clearly from right to left (relative to the surface gust front), even when using the $\nabla^{4}$ scheme of XXD96 (Fig. 5c). All these points considered, we conclude that the inviscid analytic results are not being maintained by the numerical model.

Another notable result from several analytic studies (e.g., Rottman et al. 1985; X92) is that the gravity current interface must be $60^{\circ}$ from the horizontal where the current intersects the surface (assuming free-slip boundary conditions), regardless of environmental shear $\alpha$. 
Time-averaged results for $\alpha=0$ and 2.26 are shown in Figs. 7 and 8. The slope of the interface (i.e., slope of potential temperature contours) at the surface is not entirely clear for the relatively low-resolution simulations (Figs. 7a,b and 8a,b), but clearly asymptote to $60^{\circ}$ for the relatively high-resolution simulations (Figs. 7c,d and 8c,d). In the absence of shear (Fig. 7), the interface slope quickly becomes less than $60^{\circ}$ away from the surface, which is consistent with the inviscid-flow interface shape (solid contour). For the high-shear case (Fig. 8), the interface slope quickly becomes greater than $60^{\circ}$, which is consistent with Rottman et al. (1985) and Xu and Moncrieff (1994). We conclude that the $60^{\circ}$ slope occurs (as expected) in the model simulations, but that this shape is not retained for nondimensional heights greater than roughly 0.02 (i.e., $\sim 20 \mathrm{~m}$ for the scaling parameters of XXD96).

\section{Three-dimensional simulations}

Present-day computing resources now allow for threedimensional simulations of gravity currents without much effort. The primary anticipated difference between 2D and 3D simulations is a more-realistic representation of turbulence. For 2D, vortex stretching is not possible, with a typical consequence being turbulence that grows unnaturally upscale (e.g., Moeng et al. 2004). This interpretation is supported by a comparison of snapshots from our 2D and 3D simulations (Figs. 9 and 10). The turbulent eddies are clearly larger in $2 \mathrm{D}$, especially away from the surface $(z / H \geqslant 0.2)$.

Despite the differences in turbulence structure, the model-produced propagation speeds are practically the same in our 2D and 3D simulations, regardless of resolution. All of our simulated gravity currents propagate slightly slower than the inviscid analytic results. We show nondimensional propagation speed $c_{0} / U$ over time from our highest-resolution 3D simulations $(\Delta x=\Delta y=$ $\Delta z=6.25 \mathrm{~m}$ ) in Fig. 11, where we determine $c_{0}$ by tracking the surface gust front at the lowest model level. All simulations undergo an adjustment period in the first $\sim 5$ min, likely because of the somewhat arbitrary initial interface shape (Fig. 4) for which flow cannot remain steady. For $t>10 \mathrm{~min}$ the propagation speeds (solid lines in Fig. 11) are approximately steady.

Time series of diagnosed current depth $h / H$ are shown in Fig. 12 where the solid line is the average value and gray shading illustrates minimum and maximum values from any individual gridpoint. Values for Fig. 12 are determined at some location behind the surface gust front where the flow is hydrostatic, and viscous dissipation is negligible, so a fair comparison can be made with analytic theory. After the initial adjustment, which takes $\sim 15 \mathrm{~min}$, the mean current depth becomes quasi steady.
To be clear, we note that maximum and minimum values in Fig. 12 are primarily associated with localized, transient turbulent eddies [see, e.g., Figs. 9 and 10 herein and Fig. 10 of Rotunno et al. (2011)].

As with 2D simulations, the most significant quantitative difference between simulations and inviscid analytic theory is the gravity current depth $h / H$. Specifically, values of $h / H$ from numerical simulations (for $t>$ $20 \mathrm{~min}$ ) are $0.10 \mathrm{H}-0.15 \mathrm{H}$ lower than values from inviscid analytic theory (i.e., $10 \%-40 \%$ lower than the inviscid values). This difference seems likely attributable to dissipation associated with turbulence, which is inevitable and persistent in all our simulations (2D and 3D). Time-averaged plots of dissipation rate $\epsilon$ are shown in Fig. 13, where this variable is calculated as follows:

$$
\epsilon=\tau_{i j} \frac{\partial u_{i}}{\partial x_{j}}
$$

for $i=1,2,3$ (the three coordinate dimensions), and the stress can be expressed as

$$
\tau_{i j}=\gamma \operatorname{sign}\left(1, u_{j}\right) \frac{F\left(u_{i}\right)}{\Delta},
$$

where $\gamma=u_{j} \Delta / 60$ is the flow-dependent diffusion coefficient, $\Delta$ is the grid spacing, and $F(\cdot)$ represents the diffusive flux that is implicit in this model's fifth-order advection scheme; the exact form of $F(\cdot)$ is given by Wicker and Skamarock [2002, p. 2089, their Eq. (4c)]. We note that dissipation occurs primarily in a thin layer near the diagnosed current depth (denoted by the dashed red line in Fig. 13). The maximum value of (averaged) $\epsilon$ occurs close to the leading edge of the gravity current (i.e., $x / H>-1$ ). Also, of particular relevance for analytic studies that account for dissipation, we note that $\epsilon$ is found primarily within receding flow- that is, where flow is from right to left (relative to the surface current).

\section{Analytic theory with dissipation}

The most notable result from the preceding analysis of model simulations is that the current depths are always lower than the inviscid analytic solutions. The same result was found by XXD96 for analogous simulations although, as noted above, our simulations produce systematically lower current depths than their simulations, presumably because of different numerical techniques. Because turbulence (and associated dissipation) is prevalent in our simulations, it seems clear that a proper theoretical description of these flows should include dissipation.

Dissipation in analytic models of gravity currents is notoriously complicated, primarily because there is no 

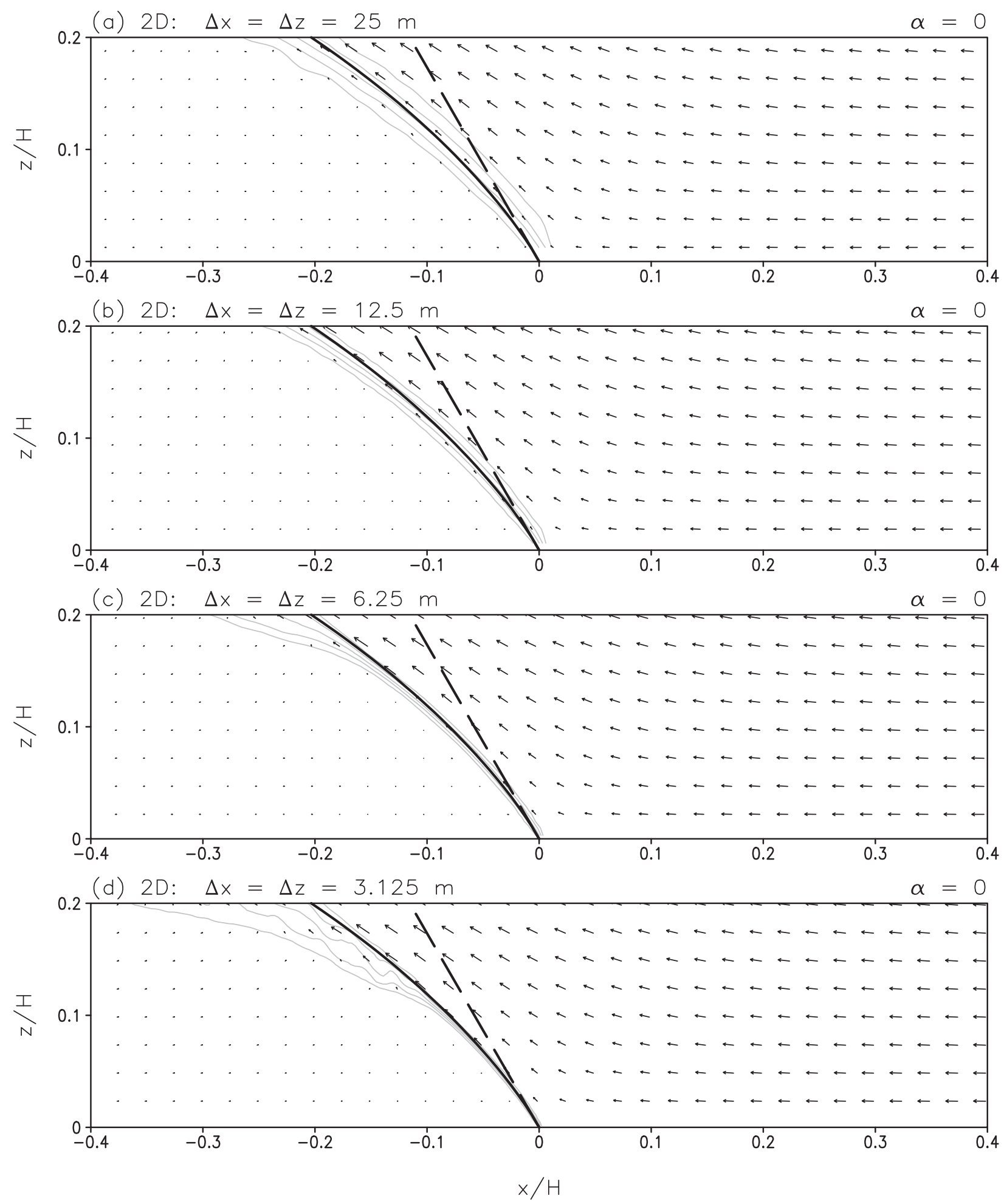

FIG. 7. Close-up view of 2D numerical simulations near the surface. Vectors and potential temperature contours are as in Fig. 5. The solid black contour is the analytic interface for steady inviscid flow. The dashed line denotes a $60^{\circ}$ angle from the horizontal. 

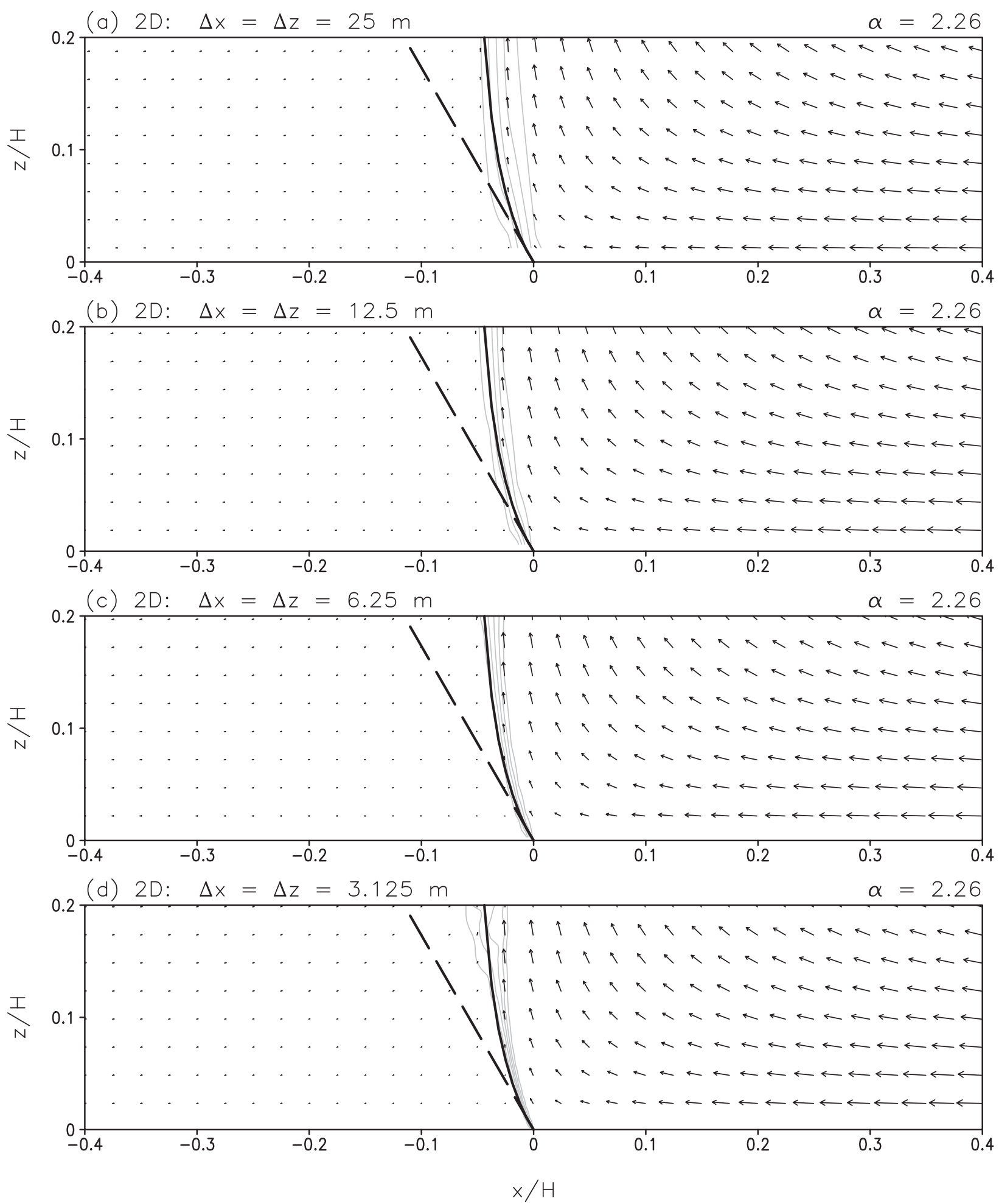

FIG. 8. As in Fig. 7, but for $\alpha=2.26$. 

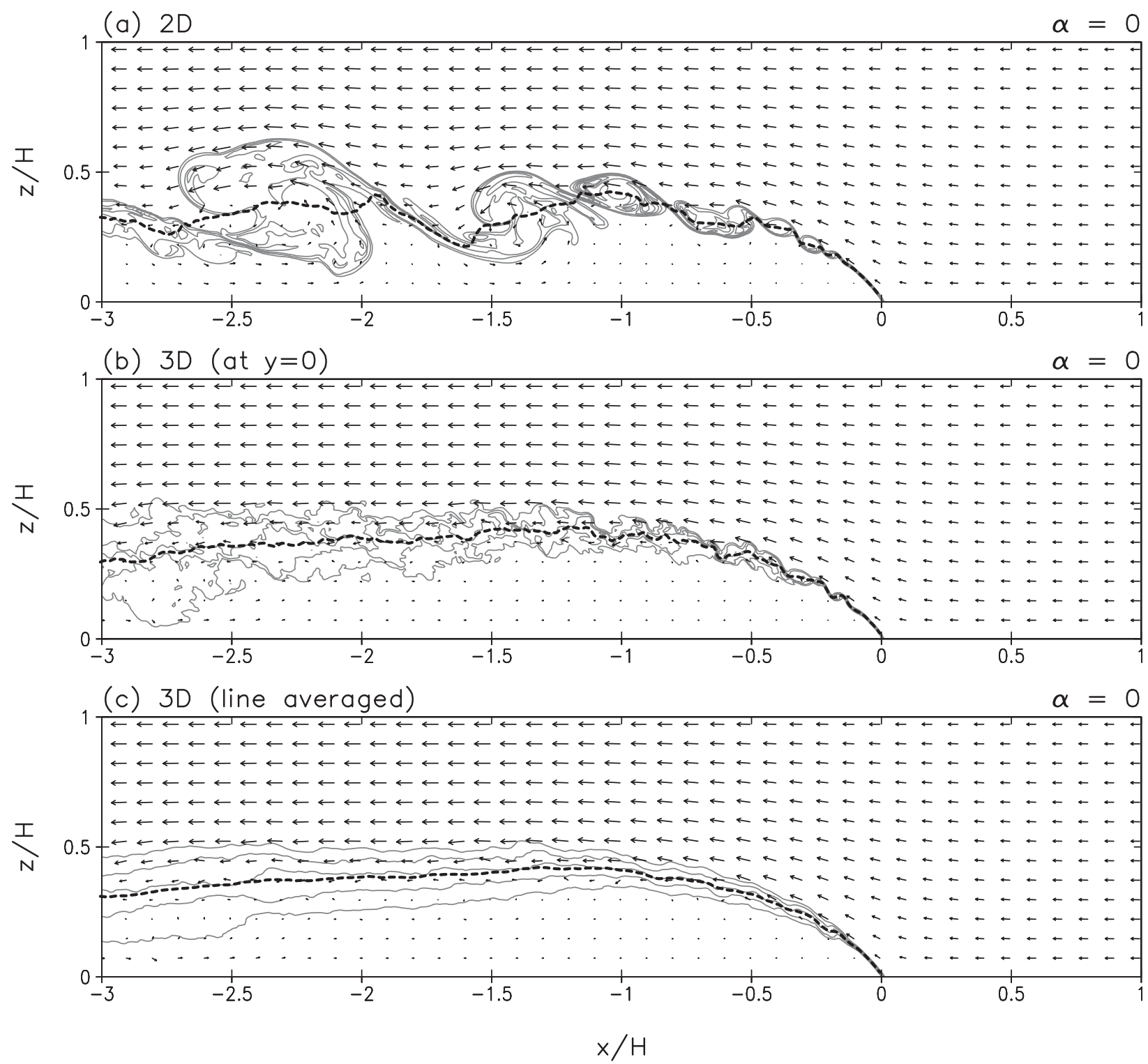

FIG. 9. As in Fig. 5, except at $t=20$ min using 6.25-m grid spacing for (a) a two-dimensional simulation, (b) a three-dimensional simulation (showing instantaneous fields at the first grid row), and (c) a three-dimensional simulation (showing fields averaged in $y$ ). The diagnosed gravity current depths (black dashed contours) in this figure are instantaneous values at $t=20 \mathrm{~min}$.

unique way to include viscous effects. For the zero-shear case, Benjamin (1968) assumed that the downstream (i.e., receding) flow remained unsheared, which is equivalent to assuming the dissipation occurs uniformly with height. Although dissipation is actually localized near the current interface in realistic gravity currents, which Benjamin acknowledged (p. 220), his assumption of uniform dissipation with height simplifies the mathematics considerably and yields results (such as the well-known $h / H=0.347$ depth for maximum possible dissipation rate) that subsequent studies have shown to be qualitatively accurate and useful in practice (e.g., Klemp et al.
1994). Other approaches to include dissipation are certainly possible; for example, Klemp et al. (1994; see their appendix) allowed for localized dissipation near the current interface, but found that results are "nearly indistinguishable" from Benjamin's results.

For sheared environments, the only analytic study with dissipation (to our knowledge) is X92's (see his section 4). He considered dissipation in a comprehensive manner by including two additional parameters: a magnitude of energy loss $\epsilon_{0}$ and a vorticity generation parameter $\Delta \alpha$, which essentially accounts for diffusion of vorticity between the lowest streamline (i.e., that 

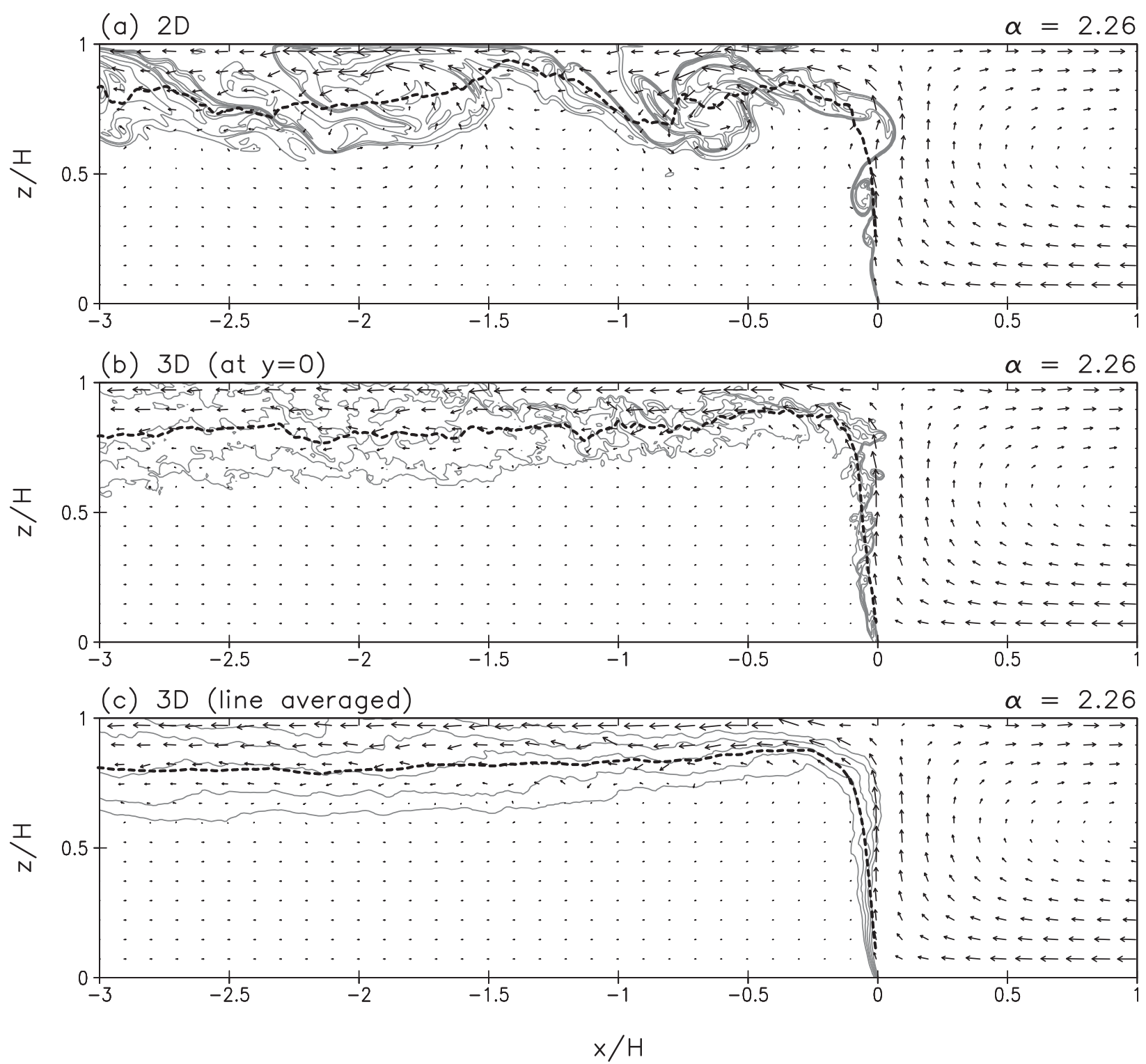

FIG. 10. As in Fig. 9, but for $\alpha=2.26$.

passing along the top of the cold air) and the inviscid flow above. Constraints imposed by flow-force balance provide closure to this framework and allow for derivation of additional parameters, like the depth $\Delta h$ of the interfacial layer where diffusion of vorticity occurs. Benjamin's approach (i.e., uniform energy loss with height) exists in X92's framework and corresponds to $\Delta \alpha=0$. (X92, p. 518, proved that uniform energy loss is only possible in this framework when $\Delta \alpha=0$.)

X92's approach allows for realism (e.g., dissipation confined near the current interface) and flexibility (in the sense that different flow structures are permitted, which in principle could be matched to simulated gravity current structure). However, we decided not to explore the complete range of possible solutions in his framework, but opted to examine only the case of $\Delta \alpha=0$ (i.e., the case of uniform energy loss) in the spirit of Benjamin's study. Although Benjamin (1968, p. 220) clearly notes the shortcomings of this approach, primarily because dissipation is usually confined near the gravity current interface (e.g., Fig. 13 herein), nevertheless, in the spirit of Klemp et al. (1994), we find results to be encouragingly close to our model simulations, as demonstrated below.

Here, we briefly review a derivation of this limiting case $(\Delta \alpha=0)$, focusing on some dynamical aspects of 

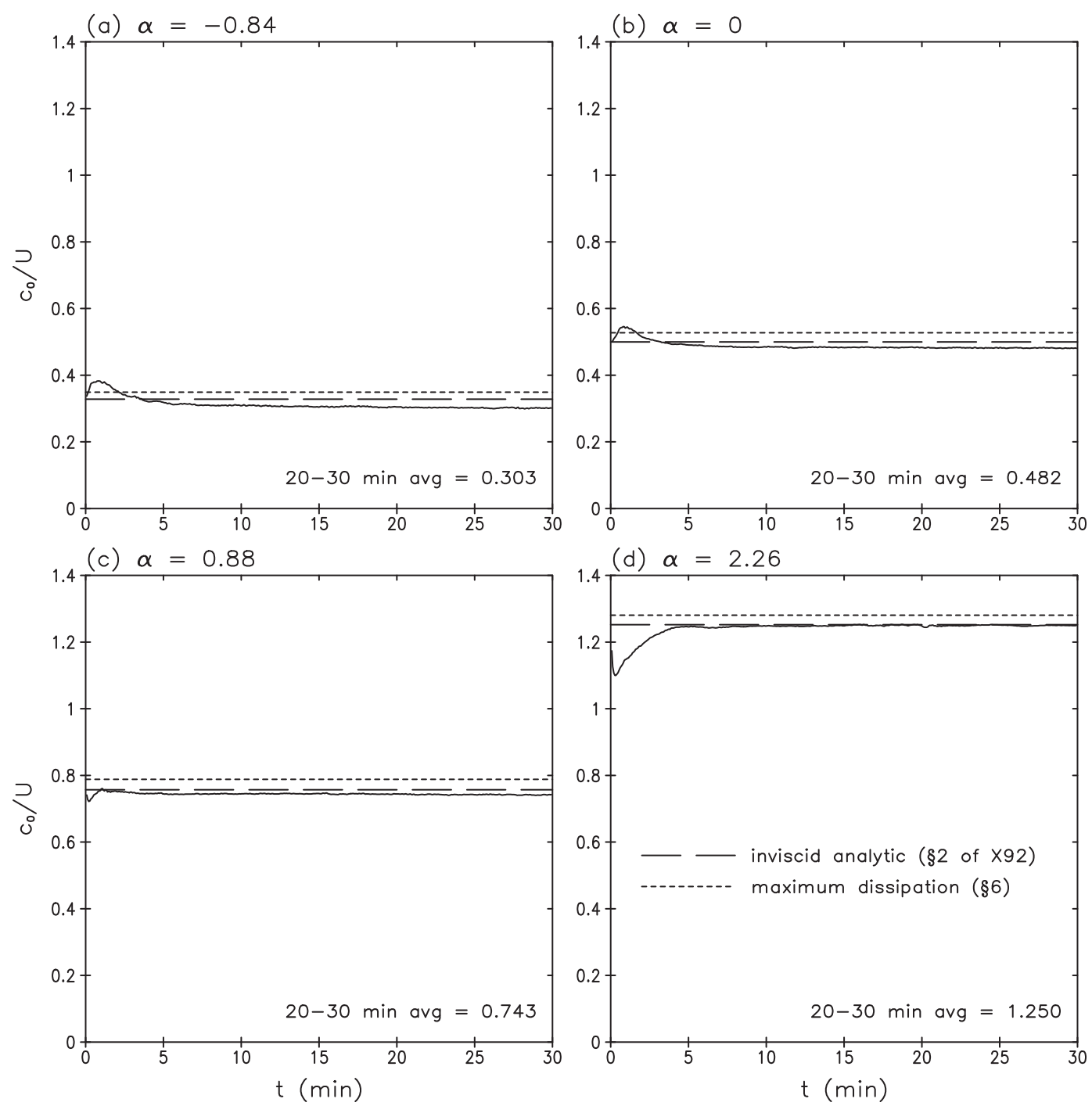

FIG. 11. Nondimensional gravity current propagation speed $\left(c_{0} / U\right.$ ) for $\alpha=$ (a) -0.84 , (b) 0 , (c) 0.88 , and (d) 2.26 . The solid line is the value from 3D simulations with $6.25-\mathrm{m}$ grid spacing. The long-dashed line denotes the (constant) analytic value for steady inviscid flow (section 2 of X92), and the short-dashed line denotes the (constant) analytic value for maximum dissipation rate (see section 6 herein).

the derivation; further details are available in X92's section 4 . The velocity profile on the right side of a control-volume $c(z)$, and that on the left side $u(z)$, are given simply by

$$
\begin{aligned}
c(z) & =-c_{0}+\alpha z \text { and } \\
u(z) & =-u_{0}+\alpha(z-h) \text { for } z \geq h \\
& =0 \text { for } z<h,
\end{aligned}
$$

where $c_{0}$ and $u_{0}$ represent front-relative flow at the bottom of the respective layers. To be clear, even though shear (i.e., vorticity) is the same in the inflow and outflow layers, vorticity conservation is not assumed along streamlines. Dissipation and diffusion processes are permitted, and assumed to occur, inside the control volume. The assumption of identical shear (vorticity) profiles is made for the sake of simplicity, and we evaluate the merits of the subsequent results below.

Flow-force balance is done in the usual way (e.g., Benjamin 1968; X92) using the conceptual setup as shown in Fig. 1, except now it is recognized that dissipation occurs somewhere inside the control volume (and, incidentally, the current interface is not smooth). We use the governing equations and methodology from section 2 of BR08 except that the incompressible approximation is made here by setting density to a constant value. 

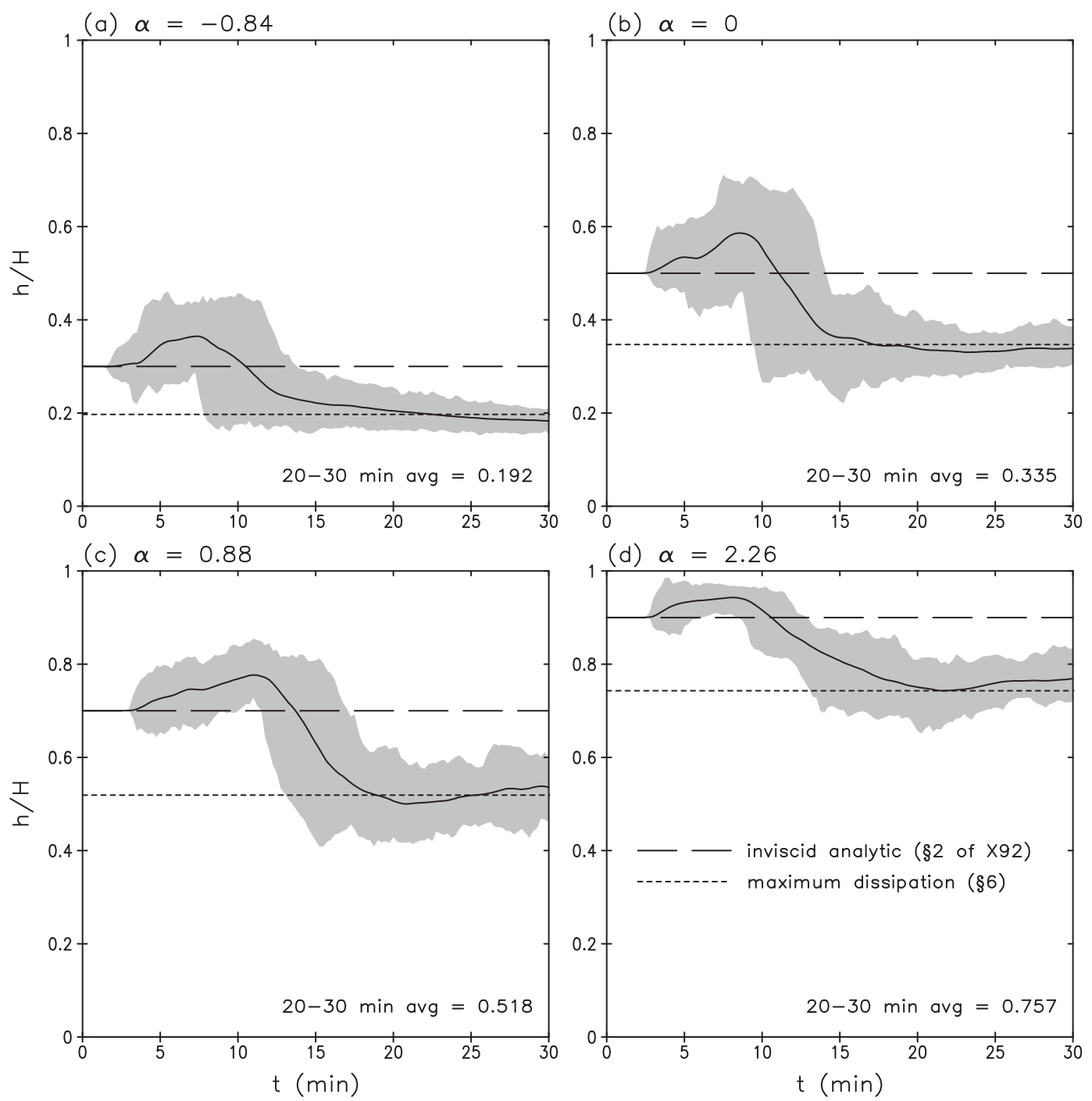

FIG. 12. Nondimensional gravity current depth $(h / H)$ for $\alpha=$ (a) -0.84 , (b) 0 , (c) 0.88 , and (d) 2.26 . The solid line shows the average value, and gray shading encloses minimum and maximum values, from 3D simulations with $6.25-\mathrm{m}$ grid spacing. The long-dashed line denotes the (constant) analytic value for inviscid flow (section 2 of X92), and the short-dashed line denotes the (constant) analytical value for maximum dissipation rate (see section 6 herein).

In brief, integration of the mass continuity over the control volume, using the sheared profiles given by Eqs. (9) and (10), yields a relation between $c_{0}$ and $u_{0}$ :

$$
c_{0}=u_{0}\left(1-\frac{h}{H}\right)+\alpha \frac{h}{H}\left(H-\frac{h}{2}\right) .
$$

The vertical velocity equation is integrated vertically on the left side of the control volume to give perturbation pressure $\phi$ as a function of height. Pressure at the lowerleft corner $\phi_{L, 0}$ is equated to the surface stagnation pressure at the gravity current gust front $\phi_{s}$, which is then related to propagation speed $c_{0}$ in the usual way by considering conservation of energy along a streamline at the surface in the environment, which yields $c_{0}^{2} / 2=\phi_{s}$. These steps (see p. 538 of BR08 for more details) provide the solution for perturbation pressure $\phi$ on the left side of the control volume:

$$
\phi_{L}(z)= \begin{cases}c_{0}^{2} / 2-\hat{b} z & \text { for } \quad z<h \\ c_{0}^{2} / 2-\hat{b} h & \text { for } \quad z \geq h\end{cases}
$$

The main solution is then determined by integration of the horizontal momentum equation over the control volume as conceptualized in Fig. 1. Although viscous effects are included in the analysis, all terms with viscosity drop out early in the derivation because these 

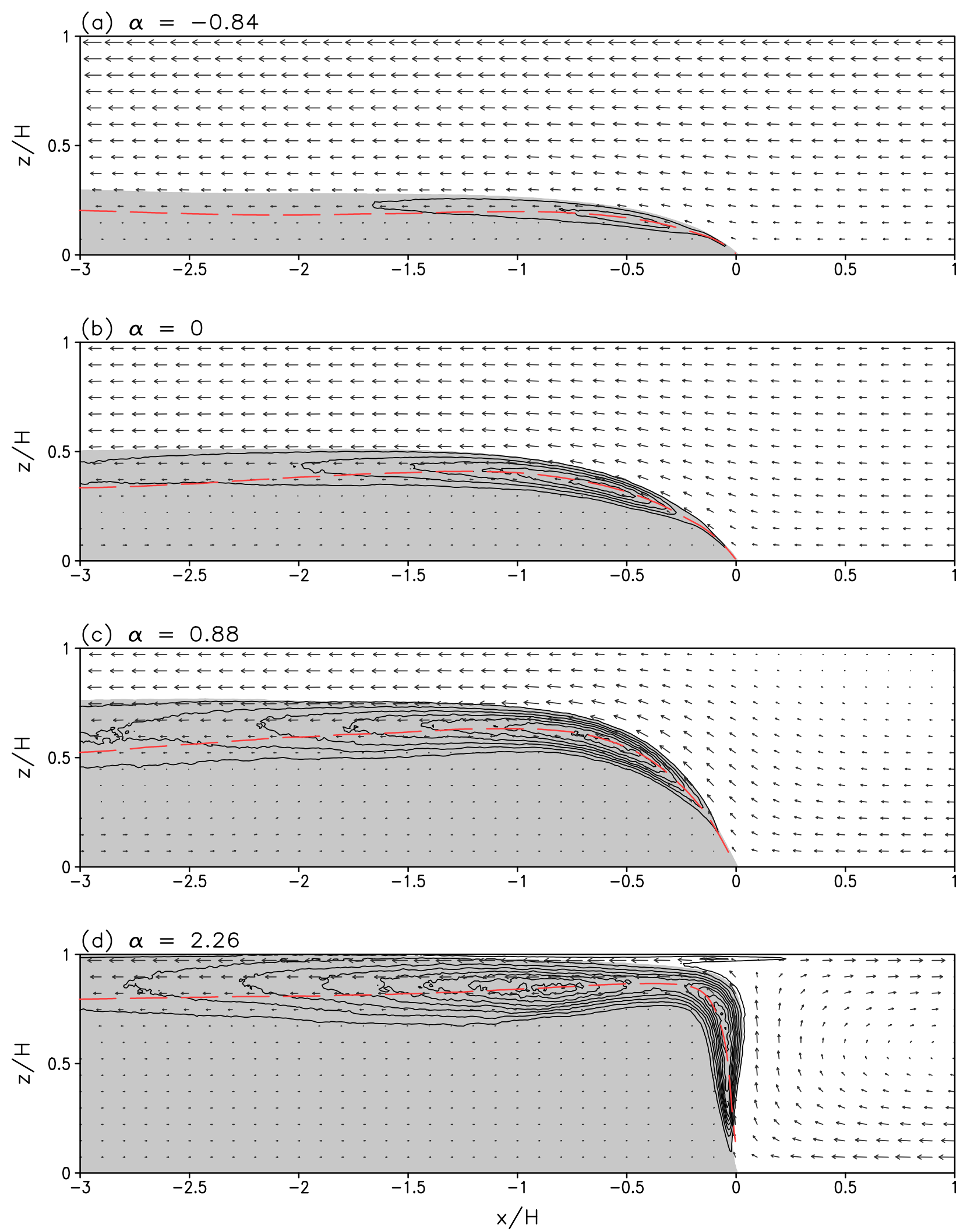

FIG. 13. Dissipation rate $\epsilon$ (contours every $0.005 \mathrm{~m}^{2} \mathrm{~s}^{-3}$ ) from 3D simulations with 6.25 -m grid spacing. Shading denotes $\theta^{\prime}<-0.3 \mathrm{~K}$. The red dashed line denotes the diagnosed gravity current depth. All fields are averaged in $y$ and then averaged in time from 20 to 30 min. 
terms are considered negligible (i.e., the flow is laminar) on the left and right boundaries of the control volume, and zero stress is assumed along the lower and upper boundaries. The remaining expression,

$$
\int_{0}^{H} c^{2} d z-\int_{h}^{H} u^{2} d z=\int_{0}^{H} \phi_{L} d z
$$

is the usual expression for flow-force balance, although here we recognize that $u$ and $c$ are functions of height. We then insert Eqs. (9), (10), and (12) into Eq. (13) and rearrange, making use of Eq. (11). A quadratic equation for $c_{0}$ can be derived, analogous to the one derived by X92 (p. 517). Expressed as $A c_{0}^{2}+B c_{0}+C=0$, we find the coefficients

$$
\begin{aligned}
A & \equiv-H(H+h), \\
B & \equiv 2 \alpha H^{2} h, \\
C & \equiv \hat{b} h(H-h)(2 H-h)-\alpha^{2} h^{2}\left(H^{2}-\frac{2}{3} h H+\frac{1}{6} h^{2}\right) .
\end{aligned}
$$

A physically realistic solution (i.e., positive propagation speed) is found for one of the roots:

$$
c_{0}=\frac{-B-\left(B^{2}-4 A C\right)^{1 / 2}}{2 A} .
$$

In contrast to inviscid-flow solutions (e.g., section 2 of X92 and section 2a herein), which permit only one value of $h / H$ for any given value of $\alpha$, analytic models with dissipation permit multiple values of gravity current depth. [See also the appendix of Klemp et al. (1994) for $\alpha=0$, and section 4b of X92 for $\alpha \neq 0$.] We summarize all possible states from the analytic solutions examined herein for four values of $\alpha$ in Fig. 14. We plot propagation speed nondimensionalized in two ways: 1) $c_{0} /(\hat{b} H)^{1 / 2}=c_{0} / U$ is plotted as a solid-black curve, which is the traditional nondimensionalization based on external parameters, and 2) $c_{0} /(\hat{b} h)^{1 / 2}$ is plotted as a solid gray curve, which readily shows whether results are finite as $h / H \rightarrow 0$ (i.e., the case of infinite channel depth). We also plot dissipation rate $e$, which is determined by the difference in energy flux between the two sides of the control volume,

$$
e \equiv \int_{0}^{H} c E_{R} d z-\int_{h}^{H} u E_{L} d z
$$

where energy on the right and left sides are, respectively, $E_{R}(z) \equiv c^{2}(z) / 2$ and $E_{L}(z) \equiv u^{2}(z) / 2+\phi_{L}(z)$. This variable is also nondimensionalized in two ways: 1) $e /\left(\hat{b}^{3} H^{5}\right)^{1 / 2}$ is plotted as a dashed black curve and
2) $e /\left(\hat{b}^{3} h^{5}\right)^{1 / 2}$ as a dashed gray curve. Steady results that require net kinetic energy input [negative values of $e$ as defined by Eq. (16)] are unphysical and are not shown in Fig. 14; these states correspond to most of the empty "white space" in Fig. 14. We further exclude solutions that permit $u(H)>0$ - that is, flow from left to right on the outflow side of the control volume (Fig. 1) - which occurs only when $\alpha>0$. This latter configuration allows for circulations above the cold pool that feature sinking air somewhere above the cold pool, which are outside the scope of the present analysis. We note that $c(H)>$ 0 is permitted - that is, flow from left to right on the inflow side of the control volume-which is consistent with ascent everywhere to the right of the gravity current (see, e.g., Fig. 2c).

We reiterate that these solutions represent a small subset of the results derived by X92. For example, the rightmost point of all curves in Fig. 14 corresponds to the inviscid solutions from section 2 of X92 (note that $e=0$ at these points), our solid-black curves are subsets of X92's Fig. 7b (if plots of his $\mathrm{Fr}_{0}$ are constructed along a constant $\alpha$ ), and our black dashed curves correspond with the energy loss $\left(\epsilon_{0}\right)$ contours in his Fig. 7a (if his $\epsilon_{0}$ is plotted as a function of $h$ along a constant $\alpha$ ). Of particular interest here, the maximum-dissipation solutions (which are discussed further below) are easily identifiable by the maximum in the black dashed curves in our plots, and can be identified by the leftmost contour for a given $\alpha$ on X92's Fig. 7. For example, for $\alpha=$ 0 (Fig. 14b) the maximum dissipation rate $e /\left(\hat{b}^{3} H^{5}\right)^{1 / 2}$ occurs for $h / H=0.347$, which is point $\mathrm{M}$ on X92's Fig. 7a.

We now consider the full range of possible solutions for a given $\alpha$. For $\alpha=0$ (Fig. 14b) our results are identical to results by Benjamin (1968) (cf. his Figs. 5-7). From Fig. 14b the following well-documented conclusions can be drawn: $0 \leq h / H \leq 0.5$ is required for steady flow, the maximum possible dissipation rate $e /\left(\hat{b}^{3} H^{5}\right)^{1 / 2}$ occurs for $h / H=0.347$ and solutions are obtained for a gravity current in an infinitely deep channel $(h / H=0)$.

For the case of negative environmental shear (Fig. 14a), steady flows are possible for $0 \leq h / H \leq 0.3$, the maximum possible dissipation rate $e /\left(\hat{b}^{3} H^{5}\right)^{1 / 2}$ occurs for $h / H=0.197$, and (like the no-shear case) nonzero results are obtained for $h / H=0$.

For the two cases of positive environmental shear (Figs. 14c,d) the maximum-dissipation solutions (peaks of black dashed curve) and inviscid-flow solutions (rightmost points on all curves) are shifted toward larger values of $h / H$ as $\alpha$ increases. But the most notable difference from the other cases is that solutions are not possible for infinitely deep domains $(h / H=0)$ for $\alpha>0$. [Infinite-depth solutions are also not possible for any 

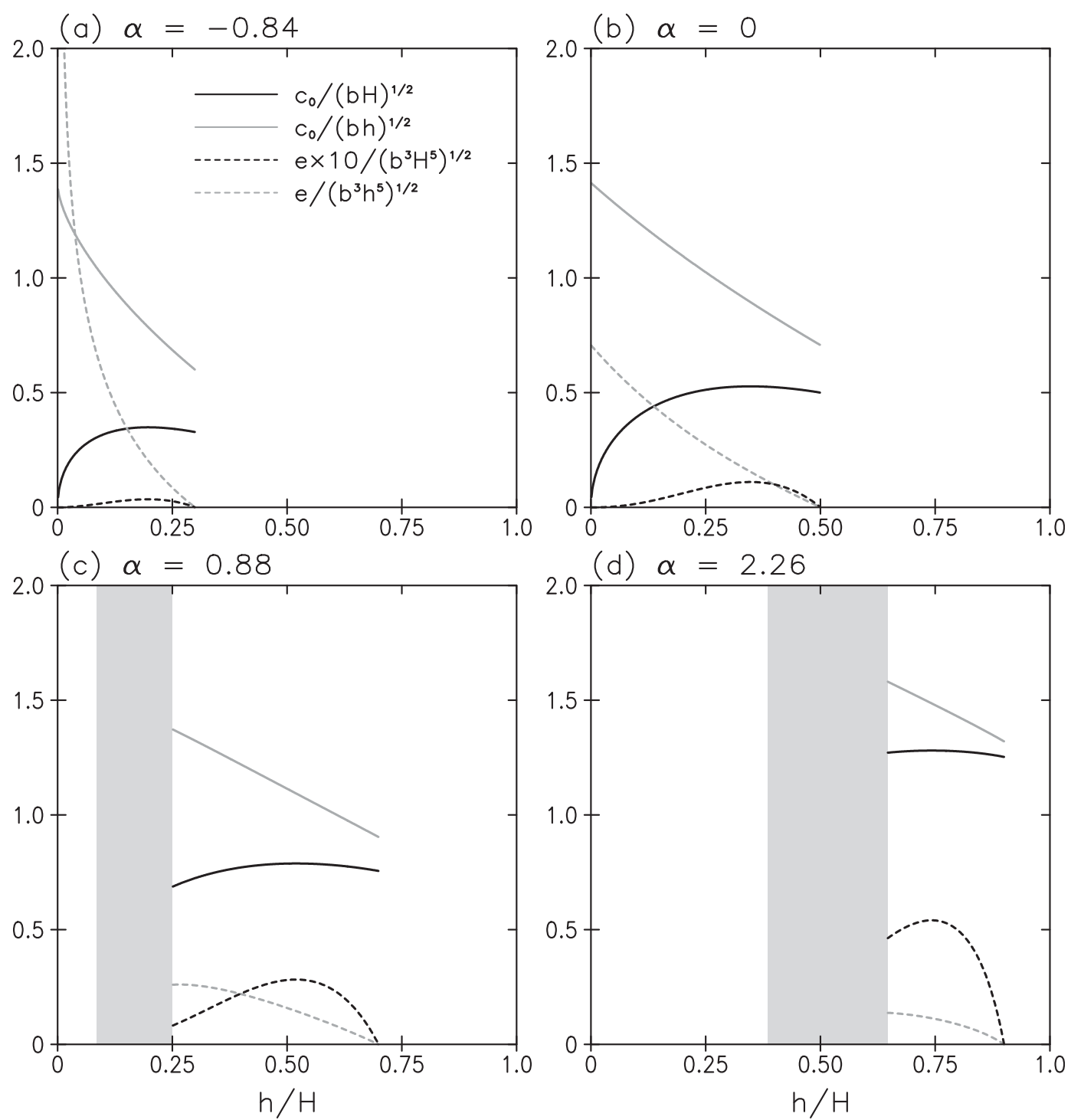

FIG. 14. Complete range of solutions for a steady analytical theory with dissipation for environmental shear $\alpha=$ (a) -0.84 , (b) 0 , (c) 0.88 , and (d) 2.26 . Solid curves show propagation speed and dashed curves show dissipation rate, both nondimensionalized in different ways, as indicated in the legend. The dashed black curve has been multiplied by 10 for clarity. The rightmost points of all results correspond to the inviscid solutions from section 2 of X92 (cf. first line of Tables 1-2); see text for explanation of how these results compare to figures in section 4 of X92. The shaded region shows where solutions are possible with $e>0$, but $u(H)>0$ (i.e., the flow is from left to right on the outflow side of Fig. 1), which is outside the scope of this study.

$u(H)>0$ (shaded region in Figs. 14c,d).] This curious result occurs for all $\alpha>0$ and is attributable to the assumption in this study that shear fills the entire channel. For an infinitely deep channel with $\alpha>0$, flow on the upper-right corner of the control volume becomes positive for $z>c_{0} / \alpha$ [from Eq. (9)]. Further, and more important, the total energy flux on the right side of the controlvolume $E_{R, t}$ [first term on right-hand side of Eq. (16)] can become positive for deep channels (i.e., small values of $h / H$ ); this situation corresponds to net energy loss through the right side of the control volume and would require net energy input within the domain to balance, which is not physically possible (under the equation set used herein). In contrast, for $\alpha \leq 0$, it is impossible to have $c>0$, and thus $E_{R, t}<0$, and so steady solutions are possible for $h / H \rightarrow 0$. We reiterate that this curious result (i.e., no solutions for infinitely deep domain with $\alpha>0$ ) is attributable to the assumption that shear fills the entire channel and is not necessarily associated with other shear profiles (e.g., shear near the surface only). 

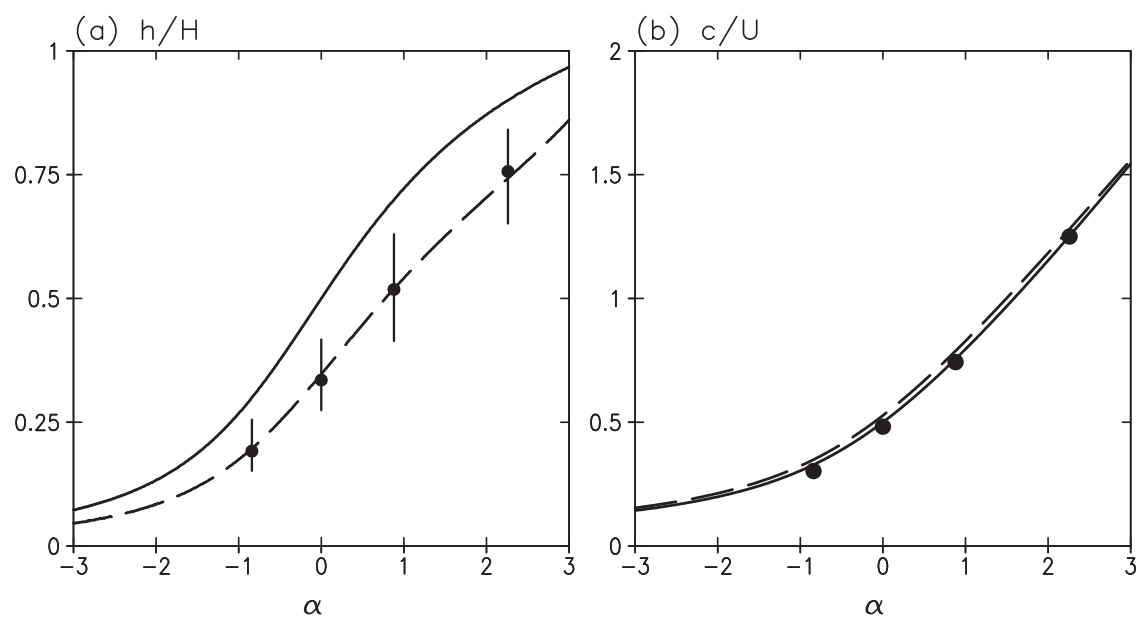

FIG. 15. Comparison of steady analytic solutions for inviscid flow (solid lines) and for maximum dissipation rate as determined in section 6 (dashed lines): (a) nondimensional current depth $h / H$; (b) nondimensional propagation speed $c / U$. See text for explanation of how these results compare with Fig. 7 of X92. Dots show average results from 3D numerical simulations with $6.25-\mathrm{m}$ grid spacing; vertical lines in (a) denote minimum and maximum values.

Results corresponding to the maximum dissipation rate [i.e., maximum value of $e /\left(\hat{b}^{3} H^{5}\right)^{1 / 2}$ ] are plotted for $-3 \leq \alpha \leq 3$ in Fig. 15 (dashed line) and are compared with inviscid analytic solutions (solid line). We note that the dashed curve in Fig. 15a connects the "turning points" of contours (i.e., "cusp points" where contours are exactly vertical) in Fig. 7a of X92, and the dashed curve in Fig. $15 \mathrm{~b}$ connects the turning points of the $\mathrm{Fr}_{0}$ contours in Fig. 7b of X92. Results from our numerical simulations (specifically our highest-resolution 3D simulations) are also included as dots. For propagation speed $c_{0} / U$ (Fig. 15b) there is a very small difference between the two analytic solutions, and the model simulations are reasonably described by either one. For current depth $h / H$ (Fig. 15a), there is a notable difference in the inviscid and maximum-dissipation solutions, although both curves have the same qualitative trend (i.e., $h / H$ increases as $\alpha$ increases). The average model results (dots) are much closer to the maximum-dissipation results. (As noted in the previous section, the higher and lower values, denoted by vertical bars in Fig. 15a, are primarily associated with transient turbulent eddies.) The better correspondence of model results with the steady maximum-dissipation analytic results supports the conclusion that dissipation plays an essential role in gravity currents [to borrow a phrase used by Benjamin (1968, p. 247)]. A similar conclusion was reached in section 5 of XXD96.

In our previous studies (BR08; Rotunno et al. 2011) we also found that simulated gravity current depths tended to be similar to the analytic maximum-dissipation value $h_{m}$, specifically for simulations in which initial cold-pool depth exceeds $h_{m}$ (as in simulations herein). This conclusion is somewhat "in the eye of the beholder" in the sense that model results do not always match perfectly (e.g., Fig. 13 of BR08), and model simulations are not precisely steady (e.g., Fig. 12 herein). To be clear, we have viewed the maximum-dissipation solutions as a likely upper bounds on steady gravity current depth rather than an expected result for any particular simulation; see BR08 (p. 543) and Rotunno et al. (2011) for further explanation.

Future work could examine analytic solutions with the more-realistic scenario in which energy dissipation is localized near the top of the cold pool (i.e., $\Delta \alpha>0$ in section 4 of X92). There are some shortcomings associated with our assumption of uniform energy loss with height, particularly for relatively deep domains ( $h / H \rightarrow 0$; see, e.g., p. 220 of Benjamin 1968). Nevertheless, the results (Fig. 15a) seem clearly superior to inviscid analytic results as descriptors of the model simulations, especially with regards to gravity current depth. We further reiterate that turbulence (and associated dissipation) is common in both observations and simulations of gravity currents (at least for high Reynolds number and Boussinesq flow), and thus some treatment of dissipation is clearly warranted in analytic studies.

\section{Summary and conclusions}

We have examined properties of gravity currents in confined channels with sheared environments, following 
the previous studies by X92 and XXD96. Steady inviscid solutions, for which the entire $2 \mathrm{D}$ flow can be determined [following Xu et al. (1992)], show that the gravity current interface just above the surface becomes steeper as environmental shear $\alpha$ increases (consistent with some results of past studies; e.g., Rottman et al. 1985, X92, and Xu and Moncrieff 1994; see section 2b herein). In an examination of a broad range of shear values, we find that interface slope can exceed $80^{\circ}$ for $\alpha>2$.

We then evaluated numerical model simulations to see whether they could reproduce the steady, inviscid analytic results. Using essentially the same initial conditions as XXD96, our 2D numerical simulations produce lower values of gravity current depth $h / H$ as compared to values from their study. We attribute this difference primarily to different numerical techniques in our numerical model that allow for a faster transition to turbulence. We also examined 3D simulations, which produce $10 \%-40 \%$ lower values of $h / H$ than steady, inviscid analytic theory.

To explain why gravity currents are always shallower in our simulations than the steady inviscid results, we reexamined the analytic flow-force balance with dissipation from section 4 of X92. We examined only the simple, but seemingly effective, assumption of uniform energy loss with height [used for the zero-shear case by Benjamin (1968)], which corresponds to $\Delta \alpha=0$ in X92's framework. We further showed that simulated current depths are similar to the value associated with maximum possible dissipation rate in this analytic framework. A primary conclusion from this comparison is that dissipation plays an important and nonnegligible role in gravity current dynamics, with or without environmental shear.

A confined channel was assumed throughout this study, which is a common assumption for studies of gravity currents. By using higher resolution, three dimensions, and by evaluating dissipative as well as inviscid analytic models, this article has broadened the perspective on this approach to studying gravity currents. However, a confined channel is generally acknowledged to be problematic when applying relatively high-shear $(\alpha \gtrsim 1)$ results to atmospheric gravity currents. XXD96 referred to the rigid upper boundary as "an important limitation" (p. 785) and noted that, for the high-shear case ( $\alpha=$ 2.26), the rigid upper boundary "clearly limit(s) the applicability of this particular experiment to atmospheric flows" (p. 779). Similarly, Davies-Jones and Markowski (2013) noted how the flow of environmental air can be affected by an "artificial lid" (p. 1214). For these reasons, we have addressed a different approach to studying gravity currents in shear in a separate study (Bryan and Rotunno 2014).
Acknowledgments. We thank Joe Klemp, Qin Xu, and an anonymous reviewer for their comments and suggestions. This study made extensive use of MUDPACK, the multigrid software for elliptic equations provided by the Computational and Information Systems Laboratory (CISL) of NCAR. We would also like to acknowledge high-performance computing support from Yellowstone (ark:/85065/d7wd3xhc), provided by NCAR/CISL, and sponsored by the National Science Foundation.

\section{REFERENCES}

Benjamin, T. B., 1968: Gravity currents and related phenomenon. J. Fluid Mech., 31, 209-248.

Bryan, G. H., and R. Rotunno, 2008: Gravity currents in a deep anelastic atmosphere. J. Atmos. Sci., 65, 536-556.

- and — 2014: The optimal state for gravity currents in shear. J. Atmos. Sci., 71, 448-468.

Crook, N. A., and M. J. Miller, 1985: A numerical and analytical study of atmospheric undular bores. Quart. J. Roy. Meteor. Soc., 111, 225-242.

Davies-Jones, R., and P. Markowski, 2013: Lifting of ambient air by density currents in sheared environments. J. Atmos. Sci., 70, 1204-1215.

Droegemeier, K. K., and R. B. Wilhelmson, 1987: Numerical simulation of thunderstorm outflow dynamics. Part I: Outflow sensitivity experiments and turbulence dynamics. J. Atmos. Sci., 44, 1180-1210.

Gal-Chen, T., and R. Somerville, 1975: On the use of a coordinate transformation for the solution of the Navier-Stokes equations. J. Comput. Phys., 17, 209-228.

Klemp, J. B., R. Rotunno, and W. C. Skamarock, 1994: On the dynamics of gravity currents in a channel. J. Fluid Mech., 269, 169-198.

Moeng, C.-H., J. C. McWilliams, R. Rotunno, P. P. Sullivan, and J. Weil, 2004: Investigating 2D modeling of atmospheric convection in the PBL. J. Atmos. Sci., 61, 889-903.

Rottman, J. W., J. C. R. Hunt, and A. Mercer, 1985: The initial and gravity-spreading phases of heavy gas dispersion: Comparison of models with phase I data. J. Hazard. Mater., 11, 261-279.

Rotunno, R., J. B. Klemp, and M. L. Weisman, 1988: A theory for strong, long-lived squall lines. J. Atmos. Sci., 45, 463-485.

,-- G. H. Bryan, and D. J. Muraki, 2011: Models of nonBoussinesq lock-exchange flow. J. Fluid Mech., 675, 1-26.

Shen, Y., and G. Zha, 2010: Improvement of the WENO scheme smoothness estimator. Int. J. Numer. Methods Fluids, 64, 653-675.

Ungarish, M., 2009: An Introduction to Gravity Currents and Intrusions. CRC Press, 489 pp.

von Kármán, T., 1940: The engineer grapples with nonlinear problems. Bull. Amer. Math. Soc., 46, 615-683.

Weisman, M. L., 1992: The role of convectively generated rearinflow jets in the evolution of long-lived mesoconvective systems. J. Atmos. Sci., 49, 1826-1847.

Wicker, L. J., and W. C. Skamarock, 2002: Time splitting methods for elastic models using forward time schemes. Mon. Wea. Rev., 130, 2088-2097.

Wyngaard, J. C., 2010: Turbulence in the Atmosphere. Cambridge University Press, $393 \mathrm{pp}$

Xu, Q., 1992: Density currents in shear flows-A two-fluid model. J. Atmos. Sci., 49, 511-524. 
— , and M. W. Moncrieff, 1994: Density current circulations in shear flows. J. Atmos. Sci., 51, 434-446.

, F.-S. Zhang, and G.-P. Lou, 1992: Finite element solutions of free-interface density currents. Mon. Wea. Rev., 120, 230-233.

, M. Xue, and K. K. Droegemeier, 1996: Numerical simulations of density currents in sheared environments within a vertically confined channel. J. Atmos. Sci., 53, 770-786.
Xue, M., 2000: Density currents in two-layer shear flows. Quart. J. Roy. Meteor. Soc., 126, 1301-1320.

- 2002: Density currents in shear flows: Effects of rigid lid and cold-pool internal circulation, and application to squall line dynamics. Quart. J. Roy. Meteor. Soc., 128, 47-73.

_, Q. Xu, and K. K. Droegemeier, 1997: A theoretical and numerical study of density currents in nonconstant shear flows. J. Atmos. Sci., 54, 1998-2019. 